\title{
On a Stationary Schrödinger Equation with Periodic Magnetic Potential
}

\author{
PASCAL BÉGOUT*AND IAN SCHINDLER* ${ }^{*} \dagger$
}

\begin{abstract}
We prove existence results for a stationary Schrödinger equation with periodic magnetic potential satisfying a local integrability condition on the whole space using a critical value function.
\end{abstract}

\section{Contents}

1 Introduction and main result 2

2 The space $H_{A, V}^{1}\left(\mathbb{R}^{N}\right)$ and an equivalent definition of $H^{1}\left(\mathbb{R}^{N}\right)$

3 The set of dislocations $\quad 15$

4 Cocompactness $r$

5 An associated critical value function and proof of the main result 21

$\begin{array}{llr}6 & \text { Applications } & 27\end{array}$

$\begin{array}{lr}\text { A Some proofs } & 28\end{array}$

B Topological vector spaces over the field of complex numbers restricted to the field of real numbers $\quad 35$

$\begin{array}{ll}\text { References } & 37\end{array}$

*Institut de Mathématiques de Toulouse \& TSE, Université Toulouse I Capitole, 1 Esplanade de l'Université, 31080 Toulouse, Cedex 06, France

*E-mail: Pascal.Begout@math.cnrs.fr

$\dagger$ E-mail: Ian.Schindler@ut-capitole.fr

Pascal Bégout and Ian Schindler acknowledge funding from ANR under grant ANR-17-EUR-0010 (Investissements d'Avenir program)

2020 Mathematics Subject Classification: 35Q55 (35A01, 35D30)

Key Words: stationary Schrödinger equation, periodic magnetic potential, weak solution, cocompactness 


\section{Introduction and main result}

We wish to investigate for which $\lambda>0$ there is a weak solution to the stationary Schrödinger equation with magnetic potential:

$$
\left\{\begin{array}{l}
(-\mathrm{i} \nabla+A)^{2} u+V(x) u=\lambda \boldsymbol{f}(x, u(x)), \text { in } \mathbb{R}^{N}, \\
u \in H_{A, V}^{1}\left(\mathbb{R}^{N}\right),
\end{array}\right.
$$

where $N \geqslant 2, A: \mathbb{R}^{N} \longrightarrow \mathbb{R}^{N}$ is the magnetic potential, $B=\operatorname{curl} A$ is the magnetic field, $V: \mathbb{R}^{N} \longrightarrow$ $\mathbb{R}, \boldsymbol{f}$ satisfy some suitable assumptions, $\lambda>0$ and

$$
H_{A, V}^{1}\left(\mathbb{R}^{N}\right) \stackrel{\text { def }}{=}\left\{u \in L^{2}\left(\mathbb{R}^{N}\right) ; V u^{2} \in L^{1}\left(\mathbb{R}^{N}\right) \text { and }(\nabla+\mathrm{i} A) u \in L^{2}\left(\mathbb{R}^{N} ; \mathbb{C}^{N}\right)\right\} .
$$

Here, $\mathrm{i}^{2}=-1$ and in what follows, unless specified, all functions are complex-valued $\left(H^{1}\left(\mathbb{R}^{N}\right)=\right.$ $H^{1}\left(\mathbb{R}^{N} ; \mathbb{C}\right), L^{p}\left(\mathbb{R}^{N}\right)=L^{p}\left(\mathbb{R}^{N} ; \mathbb{C}\right), \mathscr{D}\left(\mathbb{R}^{N}\right)=\mathscr{D}\left(\mathbb{R}^{N} ; \mathbb{C}\right)$, etc). If $N=1$ then (1.1) is equivalent to the case $A=0$. Indeed, Assume that $A, V \in L_{\text {loc }}^{1}(\mathbb{R} ; \mathbb{R})$ and $\boldsymbol{f}\left(., e^{\mathrm{i} \theta} z\right)=e^{\mathrm{i} \theta} \boldsymbol{f}(., z)$, for any $(\theta, z) \in \mathbb{R} \times \mathbb{C}$ (which is the case in this paper). Set for any $x \in \mathbb{R}, \varphi(x)=\int_{0}^{x} A(s) \mathrm{d} s$. If $u \in H_{A, V}^{1}(\mathbb{R})$ is a solution to (1.1) then by the gauge transformation $u \longmapsto v=e^{\mathrm{i} \varphi} u$, a straighforward calculation gives that $v \in H^{1}(\mathbb{R})$ is a solution to $-\Delta v+V(x) v=\lambda \boldsymbol{f}(x, v(x))$, which is (1.1) with $A=0$. We thus restrict our study to the case $N \geqslant 2$.

We make assumptions that insure the functional associated with (1.1) is invariant with respect to the transformations $\tau_{y}: u \longmapsto e^{\mathrm{i} \varphi_{y}} u(.+y)$, where $\varphi_{y}$ is defined in (3.4) and $y \in \mathbb{Z}^{N}$. In [11], the authors stated that this set of transformations was a group of dislocations as defined in [14] which is false. In Section 3 we prove (directly) that the set $D$ of such transformations is a set of dislocations permitting us to use the profile decomposition theorem [14, Theorem 3.1, p.62-63]. In Devillanova and Tintarev [4, Appendix] this was proved by embedding the set of dislocations into a group via multiplication of $\tau_{y} \tau_{z}$ by $e^{\mathrm{i} \alpha}, \alpha \in \mathbb{R}$ in such a way that the composition agrees with $\tau_{y+z}$.

Arioli and Szulkin [1] treated a similar problem with more general conditions on $V$ (the spectrum of the operator $(-\mathrm{i} \nabla+A)^{2}+V(x)$ can be negative), but they assume the Rabinowitz condition on the right hand side. We make less restrictive assumptions on the right hand side and introduce a parameter $\lambda$ and an unbounded interval $I_{\gamma} \subset(0, \infty)$ such that for almost every $\lambda \in I_{\gamma}$ there is a solution to (1.1). In [4] a magnetic Schrödinger equation with bounded non-periodic magnetic field is studied.

In Section 2 we show that if the magnetic potential $A \in L_{\mathrm{loc}}^{N}\left(\mathbb{R}^{N} ; \mathbb{R}^{N}\right)$ and $V \in L_{\mathrm{loc}}^{\frac{N}{2}}\left(\mathbb{R}^{N} ; \mathbb{R}\right)$ then $H_{A, V}^{1}\left(\mathbb{R}^{N}\right)=H^{1}\left(\mathbb{R}^{N}\right)$. In Section 3, we introduce the set of invariant dislocations acting on (1.1) and 
prove necessary results to the dislocation theorem in [14]. In Section 4 we prove a cocompactness result. In Section 5 we introduce a related critical value function the study of which allows us to obtain our main result. In Section 6 we give some examples of nonlinearities to which our result applies.

Throughout this paper, we use the following notation. We denote by $\bar{z}$ the conjugate of the complex number $z$ and by $\operatorname{Re}(z)$ its real part. For $1 \leqslant p \leqslant \infty, p^{\prime}$ denotes the conjugate of $p$ defined by $\frac{1}{p}+\frac{1}{p^{\prime}}=1$. By $\left\{Q_{j}\right\}_{j} \geqslant 1$ we will denote a countable covering of $\mathbb{R}^{N} \backslash \mathbb{Z}^{N}$ by open unit cubes, thus $\mathbb{R}^{N}=\bigcup_{j \geqslant 1} \overline{Q_{j}}$, and $Q=(0,1)^{N}$. All vectors spaces considered will be over the field $\mathbb{R}$. For a Banach space $X$ (over $\mathbb{R})$, we denote by $X^{\star} \stackrel{\text { def }}{=} \mathscr{L}(X ; \mathbb{R})$ its topological dual and by $\langle., .\rangle_{X^{\star}, X} \in \mathbb{R}$ the $X^{\star}-X$ duality product and for a Hilbert space $H$, its (real) scalar product will be denoted by $\langle., .\rangle_{H}$. In particular, for any $T \in L^{p^{\prime}}(\Omega)$ and $\varphi \in L^{p}(\Omega)$ with $1 \leqslant p<\infty,\langle T, \varphi\rangle_{L^{p^{\prime}}(\Omega), L^{p}(\Omega)}=\operatorname{Re} \int_{\Omega} T(x) \overline{\varphi(x)} \mathrm{d} x$. See Appendix B for more details. If $u \in L^{p}\left(\mathbb{R}^{N}\right)$, with $1 \leqslant p \leqslant \infty$, and if $\Omega$ is an open subset of $\mathbb{R}^{N}$, with some abuse of notation, expression $\|u\|_{L^{p}(\Omega)}$ will stand for $\left\|u_{\mid \Omega}\right\|_{L^{p}(\Omega)}$. This convention also holds for the others functional spaces. The subscript "c" on a functional space indicates that the functions have compact support. For instance, if $\Omega \subseteq \mathbb{R}^{N}$ is an open subset then $u \in L_{\mathrm{c}}^{p}(\Omega)$ means that $u \in L^{p}(\Omega)$, supp $u \subset \Omega$ and $\operatorname{supp} u$ is a compact subset of $\mathbb{R}^{N}$. For a Banach space $E$, the notation $E_{\mathrm{w}}$ designates the space $E$ endowed with the weak topology $\sigma\left(E, E^{\star}\right)$ and $E_{W \star}^{\star}$ the space $E^{\star}$ endowed

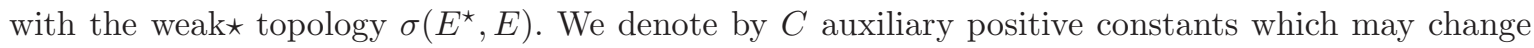
from a line to another one, and sometimes, for positive parameters $a_{1}, \ldots, a_{n}$, write $C\left(a_{1}, \ldots, a_{n}\right)$ to indicate that the constant $C$ continuously depends only on $a_{1}, \ldots, a_{n}$ (this convention also holds for constants which are not denoted by "C"). Finally, we denote by $2^{\star}=\frac{2 N}{N-2}$ the critical exponent of the embedding $H^{1}\left(\mathbb{R}^{N}\right) \hookrightarrow L^{2^{\star}}\left(\mathbb{R}^{N}\right)$, with the convention that $2^{\star}=\infty$, if $N \leqslant 2$.

We shall make the following assumptions on $A: \mathbb{R}^{N} \longrightarrow \mathbb{R}^{N}$.

Assumption 1.1. Let $\left(e_{1}, \ldots, e_{n}\right)$ be the canonical basis of $\mathbb{R}^{N}$.

1. The magnetic potential $A: \mathbb{R}^{N} \longrightarrow \mathbb{R}^{N}$ satisfies,

$$
\begin{cases}A \in L_{\text {loc }}^{N}\left(\mathbb{R}^{N} ; \mathbb{R}^{N}\right), & \text { if } N \geqslant 3, \\ A \in L_{\text {loc }}^{2+\varepsilon}\left(\mathbb{R}^{2} ; \mathbb{R}^{2}\right), \text { for some } \varepsilon>0, & \text { if } N=2 .\end{cases}
$$

and

$$
\alpha_{A} \stackrel{\text { def }}{=} \begin{cases}\sup _{j \in \mathbb{N}}\|A\|_{L^{N}\left(Q_{j}\right)}<\infty, & \text { if } N \geqslant 3, \\ \sup _{j \in \mathbb{N}}\|A\|_{L^{2+\varepsilon}\left(Q_{j}\right)}<\infty, & \text { if } N=2 .\end{cases}
$$


If $N \geqslant 3$ then there exists $\varepsilon>0$ such that,

$$
A \in L_{\mathrm{loc}}^{N+\varepsilon}\left(\mathbb{R}^{N} ; \mathbb{R}^{N}\right)
$$

2. $A$ is a $\mathbb{Z}^{N}$-periodic magnetic potential:

$$
\forall j \in \llbracket 1, N \rrbracket, \operatorname{curl} A\left(x+e_{j}\right) \stackrel{\mathscr{D}^{\prime}}{\stackrel{\left(\mathbb{R}^{N}\right)}{=}} \operatorname{curl} A(x),
$$

where $\operatorname{curl} A \in \mathscr{M}_{N}\left(\mathscr{D}^{\prime}\left(\mathbb{R}^{N} ; \mathbb{R}\right)\right)$ is the skew-symmetric, matrix-valued distribution with $A_{i j}=$ $\partial_{i} A_{j}-\partial_{j} A_{i}$.

Remark 1.2. It is easy to see that in Assumption 1.1, (1.6) is equivalent to the condition: for any $y \in \mathbb{Z}^{N}, \operatorname{curl} A(x+y) \stackrel{\mathscr{D}^{\prime}}{\stackrel{\left(\mathbb{R}^{N}\right.}{=}} \operatorname{curl} A(x)$. By Lemma 1.1 in Leinfelder [7], (1.6) is also equivalent to: for any $y \in \mathbb{Z}^{N}$, there exists $\varphi_{y} \in W_{\text {loc }}^{1, N+\varepsilon}\left(\mathbb{R}^{N} ; \mathbb{R}\right)$ such that for almost every $x \in \mathbb{R}^{N}, A(x+y)=$ $A(x)+\nabla \varphi_{y}(x)$.

Assumption 1.3. We will use the following assumptions on $V$ and $f$. Let $f: \mathbb{R}^{N} \times[0, \infty) \longrightarrow \mathbb{R}$ be such that $f(x, t)$ is measurable in $x$ and continuous in $t$ and let $F(x, t) \stackrel{\text { def }}{=} \int_{0}^{t} f(x, s) \mathrm{d} s$, for almost every $x \in \mathbb{R}^{N}$ and any $t \geqslant 0$. We extend $f$ to the complex plane by setting for almost every $x \in \mathbb{R}^{N}$ and any $z \in \mathbb{C} \backslash\{0\}, \boldsymbol{f}(x, z)=f(x,|z|) \frac{z}{|z|}$, and $\boldsymbol{f}(x, 0)=f(x, 0)$. Finally, we set for any measurable function $u: \mathbb{R}^{N} \longrightarrow \mathbb{C}$ and almost every $x \in \mathbb{R}^{N}, g(u)(x)=\boldsymbol{f}(x, u(x))$ and,

$$
\forall u \in H^{1}\left(\mathbb{R}^{N}\right), \psi(u)=\int_{\mathbb{R}^{N}} F(x,|u(x)|) \mathrm{d} x .
$$

1. For every $\varepsilon>0$, there exist $p_{\varepsilon} \in\left(2,2^{\star}\right)$ and $C_{\varepsilon}>0$ such that for almost every $x \in \mathbb{R}^{N}$ and any $t \geqslant 0$

$$
|f(x, t)| \leqslant \varepsilon\left(t+t^{2^{\star}-1}\right)+C_{\varepsilon} t^{p_{\varepsilon}-1},
$$

if $N \geqslant 3$ and

$$
|f(x, t)| \leqslant \varepsilon t+C_{\varepsilon} t^{p_{\varepsilon}-1},
$$

if $N \leqslant 2$.

$$
\exists u \in H^{1}\left(\mathbb{R}^{N}\right) \text { such that } \psi(u)>0 .
$$

2. The function $f$ and the electric potential $V: \mathbb{R}^{N} \longrightarrow \mathbb{R}$ are $\mathbb{Z}^{N}$-periodic, that is for almost every $(x, y) \in \mathbb{R}^{N} \times \mathbb{Z}^{N}$ and any $t \geqslant 0, f(x+y, t)=f(x, t)$ and $V(x+y)=V(x)$. 
3. We have,

$$
V \in L_{\mathrm{loc}}^{1}\left(\mathbb{R}^{N} ; \mathbb{R}\right) \text { and } \nu \stackrel{\text { def }}{=} \underset{x \in \mathbb{R}^{N}}{\operatorname{ess} \inf } V(x)>0 .
$$

Remark 1.4. If $N \leqslant 2$ then Assumption (1.9) is equivalent to the following:

$$
\lim _{t \rightarrow 0} \frac{f(x, t)}{t}=0, \text { uniformly in } x \in \mathbb{R}^{N},
$$

and there exist $p \in\left(2,2^{\star}\right)$ and $C>0$ such that,

$$
\text { for a.e. } x \in \mathbb{R}^{N}, \forall t \geqslant 0,|f(x, t)| \leqslant C+C t^{p-1} \text {. }
$$

If $N \geqslant 3$ then Assumption (1.8) is equivalent to (1.12), (1.13) with $p=2^{\star}$ and,

$$
\lim _{t \rightarrow \infty} \frac{f(x, t)}{t^{2^{\star}-1}}=0
$$

uniformly in $x \in \mathbb{R}^{N}$.

Assumption 1.5. The electric potential $V: \mathbb{R}^{N} \longrightarrow \mathbb{R}$ satisfies,

$$
\begin{cases}V \in L_{\mathrm{loc}}^{\frac{N}{2}}\left(\mathbb{R}^{N} ; \mathbb{R}\right) \text { and } \alpha_{V} \stackrel{\text { def }}{=} \sup _{j \in \mathbb{N}}\|V\|_{L^{\frac{N}{2}}\left(Q_{j}\right)}<\infty, & \text { if } N \geqslant 3 \\ V \in L_{\mathrm{loc}}^{1+\varepsilon}\left(\mathbb{R}^{2} ; \mathbb{R}\right) \text { and } \alpha_{V} \stackrel{\text { def }}{=} \sup _{j \in \mathbb{N}}\|V\|_{L^{1+\varepsilon}\left(Q_{j}\right)}<\infty, \text { for some } \varepsilon>0, & \text { if } N=2 .\end{cases}
$$

Remark 1.6. Note that if $V$ has the local integrability (1.14) and if furthermore $V$ is $\mathbb{Z}^{N}$-periodic then we necessarily have $\alpha_{V}<\infty$ (since $\left.\alpha_{V}=\|V\|_{L^{1}(Q)}\right)$.

Notation 1.7. Let $A$ and $V$ satisfying (1.3)-(1.4) and (1.11), respectively, and let $H_{A, V}^{1}\left(\mathbb{R}^{N}\right)$ be defined by (1.2). We shall denote by $H_{A, V}^{-1}\left(\mathbb{R}^{N}\right)$ the topological dual of the space $H_{A, V}^{1}\left(\mathbb{R}^{N}\right)$. This dual space is identified with a real vector subspace of the space of distributions $\mathscr{D}^{\prime}\left(\mathbb{R}^{N}\right)$ (see Theorem 2.3 below).

Definition 1.8. Let $A$ and $V$ satisfying (1.3)-(1.4) and (1.11), respectively, and let $H_{A, V}^{1}\left(\mathbb{R}^{N}\right)$ be defined by (1.2). We shall write that $u$ is a weak solution of (1.1) if $u \in H_{A, V}^{1}\left(\mathbb{R}^{N}\right)$ and if $u$ satisfies (1.1) in $H_{A, V}^{-1}\left(\mathbb{R}^{N}\right)$.

Remark 1.9. With respect to Definition 1.8 we note:

1. If $u \in H^{1}\left(\mathbb{R}^{N}\right)$ then $(-\mathrm{i} \nabla+A)^{2} u \in H^{-1}\left(\mathbb{R}^{N}\right)$ and,

$$
\begin{aligned}
& (-\mathrm{i} \nabla+A)^{2} u=-\Delta u-\mathrm{i} \nabla \cdot(A u)-\mathrm{i} A \cdot \nabla u+|A|^{2} u, \text { in } H^{-1}\left(\mathbb{R}^{N}\right), \\
& \langle\mathrm{i} A . \nabla u, v\rangle_{H^{-1}\left(\mathbb{R}^{N}\right), H^{1}\left(\mathbb{R}^{N}\right)}=\langle\mathrm{i} \nabla u, A v\rangle_{L^{2}\left(\mathbb{R}^{N}\right), L^{2}\left(\mathbb{R}^{N}\right),}, \\
& \left\langle|A|^{2} u, v\right\rangle_{H^{-1}\left(\mathbb{R}^{N}\right), H^{1}\left(\mathbb{R}^{N}\right)}=\langle A u, A v\rangle_{L^{2}\left(\mathbb{R}^{N}\right), L^{2}\left(\mathbb{R}^{N}\right),}
\end{aligned}
$$


for any $v \in H^{1}\left(\mathbb{R}^{N}\right)$. Indeed, if $u \in H^{1}\left(\mathbb{R}^{N}\right)$ then by Lemma 2.13 below, $A u \in L^{2}\left(\mathbb{R}^{N} ; \mathbb{C}^{N}\right)$ so that $-\Delta u \in H^{-1}\left(\mathbb{R}^{N}\right)$ and $\nabla .(A u) \in H^{-1}\left(\mathbb{R}^{N}\right)$. In addition, by Hölder's inequality, $A . \nabla u,|A|^{2} u \in$ $L_{\text {loc }}^{1}\left(\mathbb{R}^{N}\right) \hookrightarrow \mathscr{D}^{\prime}\left(\mathbb{R}^{N}\right)$ and for any $\varphi \in \mathscr{D}\left(\mathbb{R}^{N}\right)$,

$$
\langle\mathrm{i} A . \nabla u, \varphi\rangle_{\mathscr{D}^{\prime}, \mathscr{D}}=\langle\mathrm{i} \nabla u, A \varphi\rangle_{L^{2}, L^{2}} \text { and }\left\langle|A|^{2} u, \varphi\right\rangle_{\mathscr{D}^{\prime}, \mathscr{D}}=\langle A u, A \varphi\rangle_{L^{2} L^{2}} .
$$

By density and estimates in Property 1 of Lemma 2.14 below, it follows that $A . \nabla u \in H^{-1}\left(\mathbb{R}^{N}\right)$, $|A|^{2} u \in H^{-1}\left(\mathbb{R}^{N}\right)$ and (1.15)-(1.17) follow.

2. Let $u \in H_{A, V}^{1}\left(\mathbb{R}^{N}\right)$. Let $R>0$. We have,

$$
\int_{B(0, R)}|V u| \mathrm{d} x \leqslant \int_{B(0, R) \cap\{|u| \leqslant 1\}}|V||u| \mathrm{d} x+\int_{\{|u|>1\}}|V||u|^{2} \mathrm{~d} x<\infty,
$$

since $V \in L_{\text {loc }}^{1}\left(\mathbb{R}^{N} ; \mathbb{R}\right)$ and $V u^{2} \in L^{1}\left(\mathbb{R}^{N}\right)$. It follows that $V u \in L_{\text {loc }}^{1}\left(\mathbb{R}^{N}\right) \hookrightarrow \mathscr{D}^{\prime}\left(\mathbb{R}^{N}\right)$ and for any $\varphi \in \mathscr{D}\left(\mathbb{R}^{N}\right)$,

$$
\begin{aligned}
& \langle V u, \varphi\rangle_{\mathscr{D}^{\prime}, \mathscr{D}}=\operatorname{Re} \int_{\mathbb{R}^{N}} V u \bar{\varphi} \mathrm{d} x, \\
& \left|\langle V u, \varphi\rangle_{\mathscr{D}^{\prime}, \mathscr{D}}\right| \leqslant\|\sqrt{V} u\|_{L^{2}}\|\sqrt{V} \varphi\|_{L^{2}} \leqslant\|u\|_{H_{A, V}^{1}}\|\varphi\|_{H_{A, V}^{1}},
\end{aligned}
$$

by the Cauchy-Schwarz inequality (see Definition 2.1 below for the definition of $\|\cdot\|_{H_{A, V}^{1}}$ ). By the density of $\mathscr{D}\left(\mathbb{R}^{N}\right)$ in $H_{A, V}^{1}\left(\mathbb{R}^{N}\right)$ (Theorem 2.3 below), it follows that $V u \in H_{A, V}^{-1}\left(\mathbb{R}^{N}\right)$ and for any $v \in H_{A, V}^{1}\left(\mathbb{R}^{N}\right)$,

$$
\langle V u, v\rangle_{H_{A, V}^{-1}\left(\mathbb{R}^{N}\right), H_{A, V}^{1}\left(\mathbb{R}^{N}\right)}=\operatorname{Re} \int_{\mathbb{R}^{N}} V u \bar{v} \mathrm{~d} x .
$$

Finally, by Proposition 5.1 below, $g(u) \in H^{-1}\left(\mathbb{R}^{N}\right)$. In conclusion, since $H^{-1}\left(\mathbb{R}^{N}\right) \hookrightarrow H_{A, V}^{-1}\left(\mathbb{R}^{N}\right)$ (Theorem 2.3 below), it follows from (1.15) and (1.18) that

$$
(-\mathrm{i} \nabla+A)^{2} u \in H_{A, V}^{-1}\left(\mathbb{R}^{N}\right), V u \in H_{A, V}^{-1}\left(\mathbb{R}^{N}\right) \text { and } g(u) \in H_{A, V}^{-1}\left(\mathbb{R}^{N}\right) .
$$

Thus Definition 1.8 makes sense.

Our main result follows.

Theorem 1.10. Let $N \geqslant 2$ and let Assumptions 1.1 and 1.3 be satisfied. Let $H_{A, V}^{1}\left(\mathbb{R}^{N}\right)$ be defined by (1.2). Then for almost every $\lambda>0$ sufficiently large, there exists, at least one non zero weak solution to,

$$
\left\{\begin{array}{l}
-\Delta_{A} u+V(x) u=\lambda g(u) \text { in } \mathbb{R}^{N}, \\
u \in H_{A, V}^{1}\left(\mathbb{R}^{N}\right),
\end{array}\right.
$$

where $-\Delta_{A} u=(-\mathrm{i} \nabla+A)^{2} u$. 


\section{The space $H_{A, V}^{1}\left(\mathbb{R}^{N}\right)$ and an equivalent definition of $\boldsymbol{H}^{1}\left(\mathbb{R}^{N}\right)$}

In this section, we study the $H_{A, V}^{1}\left(\mathbb{R}^{N}\right)$, including the one-dimensional case $N=1$ because we believe that it is of interest for itself. For $N=1$, the corresponding assumptions to (1.4) and (1.14) are

$$
\begin{aligned}
& A \in L_{\mathrm{loc}}^{2}(\mathbb{R} ; \mathbb{R}) \text { and } \alpha_{A} \stackrel{\text { def }}{=} \sup _{j \in \mathbb{N}}\|A\|_{L^{2}\left(Q_{j}\right)}<\infty, \\
& V \in L_{\mathrm{loc}}^{1}(\mathbb{R} ; \mathbb{R}) \text { and } \alpha_{V} \stackrel{\text { def }}{=} \sup _{j \in \mathbb{N}}\|V\|_{L^{1}\left(Q_{j}\right)}<\infty,
\end{aligned}
$$

respectively.

Definition 2.1. Let $N \geqslant 1$ and let $A \in L_{\text {loc }}^{2}\left(\mathbb{R}^{N} ; \mathbb{R}^{N}\right)$ and $V \in L_{\text {loc }}^{1}\left(\mathbb{R}^{N} ; \mathbb{R}\right)$ satisfy (1.3) and (1.11), respectively. We recall that $H_{A, V}^{1}\left(\mathbb{R}^{N}\right)$ is defined by,

$$
H_{A, V}^{1}\left(\mathbb{R}^{N}\right)=\left\{u \in L^{2}\left(\mathbb{R}^{N}\right) ; V u^{2} \in L^{1}\left(\mathbb{R}^{N}\right) \text { and } \nabla_{A} u \in L^{2}\left(\mathbb{R}^{N} ; \mathbb{C}^{N}\right)\right\} .
$$

where $\nabla_{A} u=(\nabla+\mathrm{i} A) u$. We endow $H_{A, V}^{1}\left(\mathbb{R}^{N}\right)$ with the following scalar product and its corresponding norm,

$$
\begin{gathered}
\forall u, v \in H_{A, V}^{1}\left(\mathbb{R}^{N}\right),\langle u, v\rangle_{H_{A, V}^{1}\left(\mathbb{R}^{N}\right)}=\operatorname{Re} \int_{\mathbb{R}^{N}} V u \bar{v} \mathrm{~d} x+\operatorname{Re} \int_{\mathbb{R}^{N}} \nabla_{A} u \cdot \overline{\nabla_{A} v} \mathrm{~d} x, \\
\forall u \in H_{A, V}^{1}\left(\mathbb{R}^{N}\right),\|u\|_{H_{A, V}^{1}\left(\mathbb{R}^{N}\right)}^{2}=\langle u, u\rangle_{H_{A, V}^{1}\left(\mathbb{R}^{N}\right)}=\int_{\mathbb{R}^{N}} V|u|^{2} \mathrm{~d} x+\left\|\nabla_{A} u\right\|_{L^{2}\left(\mathbb{R}^{N}\right)}^{2},
\end{gathered}
$$

making this space a real pre-Hilbert space. Indeed, it follows from (1.11) that $\langle., \cdot\rangle_{H_{A, V}^{1}\left(\mathbb{R}^{N}\right)}$ is a bilinear symmetric positive definite form on $H_{A, V}^{1}\left(\mathbb{R}^{N}\right) \times H_{A, V}^{1}\left(\mathbb{R}^{N}\right)$.

Remark 2.2. Below are some comments about the definition of $H_{A, V}^{1}\left(\mathbb{R}^{N}\right)$.

1. If $u \in H_{A, V}^{1}\left(\mathbb{R}^{N}\right)$ then $\nabla_{A} u \stackrel{\text { def }}{=}(\nabla+\mathrm{i} A) u \in L^{2}\left(\mathbb{R}^{N} ; \mathbb{C}^{N}\right)$ but, a priori, we do not assume that $\nabla u$ or $A u$ belong separately in $L^{2}\left(\mathbb{R}^{N}\right)$.

2. Frequently, in the literature (see for instance Sections 7.19-7.22, p.191-195, of Lieb and Loss [8]), it is assumed that $A \in L_{\text {loc }}^{2}\left(\mathbb{R}^{N} ; \mathbb{R}^{N}\right)$ rather than $A \in L_{\text {loc }}^{N}\left(\mathbb{R}^{N} ; \mathbb{R}^{N}\right)$ and $V \equiv 1$. With these assumptions it can be shown that $H_{A, 1}^{1}\left(\mathbb{R}^{N}\right)$ is a Hilbert space having $\mathscr{D}\left(\mathbb{R}^{N}\right)$ as a dense subset. Moreover, if $u \in H_{A, 1}^{1}\left(\mathbb{R}^{N}\right)$ then $|u| \in H^{1}\left(\mathbb{R}^{N}\right)$ and the so-called diamagnetic inequality (2.11) below holds. However if $A \notin L_{\text {loc }}^{N}\left(\mathbb{R}^{N} ; \mathbb{R}^{N}\right)$ then $H^{1}\left(\mathbb{R}^{N}\right) \not \subset H_{A, 1}^{1}\left(\mathbb{R}^{N}\right)$ and $H_{A, 1}^{1}\left(\mathbb{R}^{N}\right) \not \subset H^{1}\left(\mathbb{R}^{N}\right)$. We show that if $A \in L_{\text {loc }}^{N}\left(\mathbb{R}^{N} ; \mathbb{R}^{N}\right)$ then $H_{A, 1}^{1}\left(\mathbb{R}^{N}\right)=H^{1}\left(\mathbb{R}^{N}\right)$ (see Theorem 2.5 below). 
3. Arioli and Szulkin showed (Lemma 2.3 in [1]) that if $N \geqslant 2$ and $A \in L_{\text {loc }}^{N}\left(\mathbb{R}^{N} ; \mathbb{R}^{N}\right)(A \in$ $L_{\text {loc }}^{2+\varepsilon}\left(\mathbb{R}^{N} ; \mathbb{R}^{N}\right)$, if $\left.N=2\right)$ then $H_{A, 1}^{1}(\Omega)=H^{1}(\Omega)$ with equivalent norms for open bounded subsets $\Omega$ of $\mathbb{R}^{N}$ with smooth boundaries. We extend their result to the case $\Omega=\mathbb{R}^{N}$ for any $N \geqslant 1$, under assumptions (1.3)-(1.4) (Theorem 2.5 below).

Theorem 2.3. Let $A \in L_{\mathrm{loc}}^{2}\left(\mathbb{R}^{N} ; \mathbb{R}^{N}\right)$ and $V \in L_{\mathrm{loc}}^{1}\left(\mathbb{R}^{N} ; \mathbb{R}\right)$ satisfy $(1.3)-(1.4)((2.1)$, if $N=1)$ and (1.11), respectively, and let $H_{A, V}^{1}\left(\mathbb{R}^{N}\right)$ be defined by (2.3). Then,

$$
\begin{gathered}
H_{A, V}^{1}\left(\mathbb{R}^{N}\right)=\left\{u \in H^{1}\left(\mathbb{R}^{N}\right) ; V u^{2} \in L^{1}\left(\mathbb{R}^{N}\right) \text { and } A u \in L^{2}\left(\mathbb{R}^{N} ; \mathbb{C}^{N}\right)\right\}, \\
H_{A, V}^{1}\left(\mathbb{R}^{N}\right) \text { is a separable Hilbert space, } \\
\mathscr{D}\left(\mathbb{R}^{N}\right) \hookrightarrow H_{A, V}^{1}\left(\mathbb{R}^{N}\right) \hookrightarrow H^{1}\left(\mathbb{R}^{N}\right),
\end{gathered}
$$

with both dense embeddings. In particular, each term in the integrals of $\langle., .\rangle_{H_{A, V}^{1}\left(\mathbb{R}^{N}\right)}$ belongs to $L^{1}\left(\mathbb{R}^{N}\right)$. In addition,

$$
H^{-1}\left(\mathbb{R}^{N}\right) \hookrightarrow H_{A, V}^{-1}\left(\mathbb{R}^{N}\right) \hookrightarrow \mathscr{D}^{\prime}\left(\mathbb{R}^{N}\right),
$$

where $H_{A, V}^{-1}\left(\mathbb{R}^{N}\right) \stackrel{\text { def }}{=}\left(H_{A, V}^{1}\left(\mathbb{R}^{N}\right)\right)^{\star}$ and both dense embeddings.

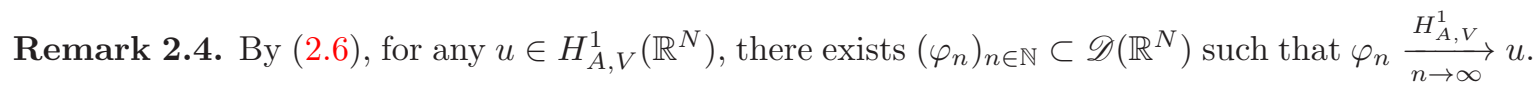
As with the classical proofs of density we have for any $n \in \mathbb{N},\left\|\varphi_{n}\right\|_{L^{p}\left(\mathbb{R}^{N}\right)} \leqslant\|u\|_{L^{p}\left(\mathbb{R}^{N}\right)}$, and $p \in[1, \infty]$, if $u \in L^{p}\left(\mathbb{R}^{N}\right)$. See Lemma 2.16 .

Theorem 2.5. Let $A \in L_{\text {loc }}^{2}\left(\mathbb{R}^{N} ; \mathbb{R}^{N}\right)$ satisfy $(1.3)-(1.4)((2.1)$, if $N=1)$ and let $V \in L_{\text {loc }}^{1}\left(\mathbb{R}^{N} ; \mathbb{R}\right)$ satisfy (1.11) and (1.14) ((1.11) and (2.2), if $N=1)$. Then,

$$
H_{A, V}^{1}\left(\mathbb{R}^{N}\right)=H^{1}\left(\mathbb{R}^{N}\right)
$$

with equivalent norms.

Remark 2.6. To find examples such that $H_{A, V}^{1}\left(\mathbb{R}^{N}\right) \subsetneq H^{1}\left(\mathbb{R}^{N}\right)$, note that by (2.15) below, assuming (1.3)-(1.4), for any $u \in H^{1}\left(\mathbb{R}^{N}\right), u \in H_{A, V}^{1}\left(\mathbb{R}^{N}\right)$ if, and only if, $V u^{2} \in L^{1}\left(\mathbb{R}^{N}\right)$. So we look for a $V$ that does not satisfy (1.14). If $N=1$ we must have $\alpha_{V}=\infty$. In other words, $V$ cannot be $\mathbb{Z}^{N}$ periodic. Below, for each $N$, we give an example of a $V$ and a $u \in H^{1}\left(\mathbb{R}^{N}\right)$ such that $u \notin H_{A, V}^{1}\left(\mathbb{R}^{N}\right)$. For $N \geqslant 2, u$ is a positive continuous function over $\mathbb{R}^{N} \backslash\{0\}$ such that for $|x|>10, u(x)=|x|^{-N}$. We give its definition for $x$ near 0 below.

1. For $N=1$. Define for any $x \in \mathbb{R}, V(x)=x^{4}+1$ and $u(x)=\left(x^{2}+1\right)^{-1}$. Then $V$ satisfies (1.11), $u \in H^{1}\left(\mathbb{R}^{N} ; \mathbb{R}\right)$ but $V u^{2} \notin L^{1}\left(\mathbb{R}^{N} ; \mathbb{R}\right)$. 
2. For $N=2$. For $|x|<e^{-e}$, let $u(x)=\ln |\ln | x||$ and let $V$ be $\mathbb{Z}^{N}$-periodic such that for any $x \in Q$,

$$
V(x)=\frac{1}{|x|^{2}|\ln | x||(\ln |\ln | x||)^{2}} \mathbb{1}_{\left\{0<|x|<e^{-e}\right\}}(x)+\mathbb{1}_{\left\{|x| \geqslant e^{-e}\right\} \cap Q}(x) .
$$

Then $V$ satisfies $(1.11)$ but for any $p \in(1, \infty], V \notin L_{\text {loc }}^{p}\left(\mathbb{R}^{2} ; \mathbb{R}\right)$, so that $V$ does not verify (1.14). In addition, $u \in H^{1}\left(\mathbb{R}^{N} ; \mathbb{R}\right)$ but $V u^{2} \notin L^{1}\left(\mathbb{R}^{N} ; \mathbb{R}\right)$.

3. For $N \geqslant 3$. Let for $|x|<1, u(x)=|x|^{-\frac{N-2}{4}}$ and let $V$ be $\mathbb{Z}^{N}$-periodic such that for any $x \in Q$, $V(x)=|x|^{-\frac{N+2}{2}}$. Then $V$ satisfies (1.11) but $V \notin L_{\text {loc }}^{\frac{N}{2}}\left(\mathbb{R}^{N} ; \mathbb{R}\right)$, so that $V$ does not verify (1.14). In addition, $u \in H^{1}\left(\mathbb{R}^{N} ; \mathbb{R}\right)$ but $V u^{2} \notin L^{1}\left(\mathbb{R}^{N} ; \mathbb{R}\right)$.

We recall that $-\Delta_{A}=(-\mathrm{i} \nabla+A)^{2}$ and $\nabla_{A}=\nabla+\mathrm{i} A$.

Theorem 2.7. Let $A \in L_{\text {loc }}^{2}\left(\mathbb{R}^{N} ; \mathbb{R}^{N}\right)$ satisfy (1.3)-(1.4) ((2.1), if $\left.N=1\right)$. If $u \in H^{1}\left(\mathbb{R}^{N}\right)$ then $-\Delta_{A} u \in H^{-1}\left(\mathbb{R}^{N}\right)$ and for any $v \in H^{1}\left(\mathbb{R}^{N}\right)$,

$$
\left\langle-\Delta_{A} u, v\right\rangle_{H^{-1}\left(\mathbb{R}^{N}\right), H^{1}\left(\mathbb{R}^{N}\right)}=\left\langle\nabla_{A} u, \nabla_{A} v\right\rangle_{L^{2}\left(\mathbb{R}^{N}\right), L^{2}\left(\mathbb{R}^{N}\right)} .
$$

If in addition $V$ satisfies (1.11) and if $H_{A, V}^{1}\left(\mathbb{R}^{N}\right)$ is defined by (2.3) then for any $T \in H^{-1}\left(\mathbb{R}^{N}\right)$, $T \in H_{A, V}^{-1}\left(\mathbb{R}^{N}\right)$ and

$$
\langle T, u\rangle_{H_{A, V}^{-1}\left(\mathbb{R}^{N}\right), H_{A, V}^{1}\left(\mathbb{R}^{N}\right)}=\langle T, u\rangle_{H^{-1}\left(\mathbb{R}^{N}\right), H^{1}\left(\mathbb{R}^{N}\right)} .
$$

for any $u \in H_{A, V}^{1}\left(\mathbb{R}^{N}\right)$,

Remark 2.8. Let $\lambda>0$ and let $u$ be a solution to (1.19). By Definition 1.8, we may take the $H_{A, V}^{-1}-H_{A, V}^{1}$ duality product of (1.19) with $u$. We have by (2.9), (2.8) and (1.18),

$$
\|u\|_{H_{A, V}^{1}\left(\mathbb{R}^{N}\right)}^{2}=\lambda\langle g(u), u\rangle_{H_{A, V}^{-1}\left(\mathbb{R}^{N}\right), H_{A, V}^{1}\left(\mathbb{R}^{N}\right)} \geqslant 0 .
$$

It follows that if $g \equiv 0$ then necessarily $u \equiv 0$. Note that if $\psi$, defined by (1.7), satisfies (1.10) then $g \not \equiv 0$.

Remark 2.9. Let $A \in L_{\text {loc }}^{2}\left(\mathbb{R}^{N} ; \mathbb{R}^{N}\right)$ satisfy (1.3)-(1.4) ((2.1), if $\left.N=1\right)$. It follows from Theorem 2.7 and (2.16) below that $-\Delta_{A} \in \mathscr{L}\left(H^{1}\left(\mathbb{R}^{N}\right) ; H^{-1}\left(\mathbb{R}^{N}\right)\right)$. If, in addition, $V \in L_{\text {loc }}^{1}\left(\mathbb{R}^{N} ; \mathbb{R}\right)$ satisfies (1.11) then by Theorems 2.3 and $2.7,-\Delta_{A \mid H_{A, V}^{1}}\left(\mathbb{R}^{N}\right) \in \mathscr{L}\left(H_{A, V}^{1}\left(\mathbb{R}^{N}\right) ; H_{A, V}^{-1}\left(\mathbb{R}^{N}\right)\right)$.

We split the proof of Theorem 2.3 in several lemmas. We begin by recalling the diamagnetic inequality for functions belonging in $H_{A, V}^{1}\left(\mathbb{R}^{N}\right)$. Its proof is well-known (Lieb and Loss [8], Theorem 7.21, p.193). For the sake of completeness, we sketch the proof. 
Theorem 2.10 (Diamagnetic inequality, [8]). Let $A \in L_{\text {loc }}^{2}\left(\mathbb{R}^{N} ; \mathbb{R}^{N}\right)$. Let $u \in L^{2}\left(\mathbb{R}^{N}\right)$ be such that $(\nabla+\mathrm{i} A) u \in L^{2}\left(\mathbb{R}^{N} ; \mathbb{C}^{N}\right)$. Then $\nabla u, A u \in L_{\text {loc }}^{1}\left(\mathbb{R}^{N} ; \mathbb{C}^{N}\right),|u| \in H^{1}\left(\mathbb{R}^{N}\right),(\nabla+\mathrm{i} A) u \stackrel{\text { a.e. }}{=} \nabla u+\mathrm{i} A u$ and

$$
|\nabla| u|| \stackrel{\text { a.e. }}{\leqslant}|\nabla u+\mathrm{i} A u| .
$$

Lemma 2.11 ([8]). If $u \in H^{1}\left(\mathbb{R}^{N}\right)$ then $|u| \in H^{1}\left(\mathbb{R}^{N} ; \mathbb{R}\right)$ and $|\nabla| u|| \stackrel{\text { a.e. }}{\leqslant}|\nabla u|$.

Proof of Theorem 2.10 and Lemma 2.11. We recall that if $u \in W_{\text {loc }}^{1, p}\left(\mathbb{R}^{N}\right)$, for some $1 \leqslant$ $p \leqslant \infty$, then $|u| \in W_{\text {loc }}^{1, p}\left(\mathbb{R}^{N} ; \mathbb{R}\right)$ and $\nabla|u| \stackrel{\text { a.e. }}{=} \operatorname{Re}\left(\frac{\bar{u}}{|u|} \nabla u\right)^{1}$ (Theorem 6.17, p.152, in Lieb and Loss [8]). This proves Lemma 2.11. Now, let $u \in L^{2}\left(\mathbb{R}^{N}\right)$ be such that $(\nabla+\mathrm{i} A) u \in L^{2}\left(\mathbb{R}^{N} ; \mathbb{C}^{N}\right)$. Then $\nabla u \in H^{-1}\left(\mathbb{R}^{N} ; \mathbb{C}^{N}\right)$ and, by the Cauchy-Schwarz inequality, $A u \in L_{\text {loc }}^{1}\left(\mathbb{R}^{N} ; \mathbb{C}^{N}\right)$. This implies that $\nabla u \in L_{\text {loc }}^{1}\left(\mathbb{R}^{N} ; \mathbb{C}^{N}\right)$. We then infer, $u \in W_{\text {loc }}^{1,1}\left(\mathbb{R}^{N}\right)$ and $(\nabla+\mathrm{i} A) u \stackrel{\text { a.e. }}{=} \nabla u+\mathrm{i} A u$. And since $\operatorname{Re}\left(\frac{\bar{u}}{|u|}(\nabla u+\mathrm{i} A u)\right) \stackrel{\text { a.e. }}{=} \operatorname{Re}\left(\frac{\bar{u}}{|u|} \nabla u\right) \stackrel{\text { a.e. }}{=} \nabla|u|$, one obtains that $|u| \in H^{1}\left(\mathbb{R}^{N}\right)$ and (2.11).

Lemma 2.12. Let $u \in L^{2}\left(\mathbb{R}^{N}\right)$ be such that $|u| \in H^{1}\left(\mathbb{R}^{N} ; \mathbb{R}\right)$.

1. If $A \in L_{\text {loc }}^{2}\left(\mathbb{R}^{N} ; \mathbb{R}^{N}\right)$ satisfies (1.3)-(1.4) ((2.1), if $\left.N=1\right)$ then $A u \in L^{2}\left(\mathbb{R}^{N} ; \mathbb{C}^{N}\right)$ and,

$$
\|A u\|_{L^{2}\left(\mathbb{R}^{N}\right)} \leqslant C \alpha_{A}\||u|\|_{H^{1}\left(\mathbb{R}^{N}\right)},
$$

where $C=C(N)(C=C(\varepsilon)$, if $N=2)$.

2. If $V$ satisfies (1.14) $((2.2)$, if $N=1)$ then $V u^{2} \in L^{1}\left(\mathbb{R}^{N}\right)$ and,

$$
\left\|V u^{2}\right\|_{L^{1}\left(\mathbb{R}^{N}\right)} \leqslant C \alpha_{V}\||u|\|_{H^{1}\left(\mathbb{R}^{N}\right)}^{2}
$$

where $C=C(N)(C=C(\varepsilon)$, if $N=2)$.

Proof. Let $u \in L^{2}\left(\mathbb{R}^{N}\right)$ be such that $|u| \in H^{1}\left(\mathbb{R}^{N} ; \mathbb{R}\right)$. We start by proving Property 1 with $N \geqslant 3$. By the Sobolev embedding $H^{1}\left(Q_{j}\right) \hookrightarrow L^{2^{\star}}\left(Q_{j}\right)$, there exists $C=C\left(N,\left|Q_{j}\right|\right)$ such that for any $j \in \mathbb{N}$, $\|u\|_{L^{2^{\star}}\left(Q_{j}\right)} \leqslant C\||u|\|_{H^{1}\left(Q_{j}\right)}$. Actually, $C$ only depends on $N$ since for any $j \in \mathbb{N},\left|Q_{j}\right|=1$. It follows from Hölder's inequality that,

$$
\begin{aligned}
\int_{\mathbb{R}^{N}}|A u|^{2} \mathrm{~d} x & =\sum_{j \in \mathbb{N}} \int_{Q_{j}}|A u|^{2} \mathrm{~d} x \\
& \leqslant \sum_{j \in \mathbb{N}}\|A\|_{L^{N}\left(Q_{j}\right)}^{2}\|u\|_{L^{2^{\star}}\left(Q_{j}\right)}^{2} \\
& \leqslant C^{2} \alpha_{A}^{2} \sum_{j \in \mathbb{N}}\||u|\|_{H^{1}\left(Q_{j}\right)}^{2} \\
& =C^{2} \alpha_{A}^{2}\||u|\|_{H^{1}\left(\mathbb{R}^{N}\right)}^{2} .
\end{aligned}
$$

\footnotetext{
${ }^{1} \nabla|u|=0$, almost everywhere where $u=0$.
} 
If $N=2$ then the second line is replaced with $\sum_{j \in \mathbb{N}}\|A\|_{L^{2+\varepsilon}\left(Q_{j}\right)}^{2}\|u\|_{L^{\frac{2(2+\varepsilon)}{\varepsilon}}\left(Q_{j}\right)}^{2}$ and we use the embed$\operatorname{ding} H^{1}\left(Q_{j}\right) \hookrightarrow L^{\frac{2(2+\varepsilon)}{\varepsilon}}\left(Q_{j}\right)$, while if $N=1$ then the second line is replaced with $\sum_{j \in \mathbb{N}}\|A\|_{L^{2}\left(Q_{j}\right)}^{2}\|u\|_{L^{\infty}\left(Q_{j}\right)}^{2}$ and we use the embedding $H^{1}\left(Q_{j}\right) \hookrightarrow L^{\infty}\left(Q_{j}\right)$. Hence 1. Property 2 follows in the same way: replace $A$ with $\sqrt{|V|}$ in the above estimates.

Lemma 2.13. Let $A \in L_{\mathrm{loc}}^{2}\left(\mathbb{R}^{N} ; \mathbb{R}^{N}\right)$ satisfy (1.3)-(1.4) ((2.1), if $\left.N=1\right)$. Then,

$$
\begin{gathered}
(\nabla+\mathrm{i} A) u \in L^{2}\left(\mathbb{R}^{N} ; \mathbb{C}^{N}\right) \Longleftrightarrow u \in H^{1}\left(\mathbb{R}^{N}\right), \\
\nabla u \in L^{2}\left(\mathbb{R}^{N} ; \mathbb{C}^{N}\right) \Longrightarrow A u \in L^{2}\left(\mathbb{R}^{N} ; \mathbb{C}^{N}\right) .
\end{gathered}
$$

Finally, if $u \in H^{1}\left(\mathbb{R}^{N}\right)$ then $(\nabla+\mathrm{i} A) u=\nabla u+\mathrm{i} A u$, in $L^{2}\left(\mathbb{R}^{N} ; \mathbb{C}^{N}\right)$ and

$$
\|\nabla u+\mathrm{i} A u\|_{L^{2}\left(\mathbb{R}^{N}\right)} \leqslant C\|u\|_{H^{1}\left(\mathbb{R}^{N}\right)}
$$

where $C=C\left(\alpha_{A}, N\right)\left(C=C\left(\alpha_{A}, N, \varepsilon\right)\right.$, if $\left.N=2\right)$.

Proof. Let $A$ satisfy (1.3)-(1.4) ((2.1), if $N=1)$. Let $u \in L^{2}\left(\mathbb{R}^{N}\right)$.

- If $\nabla u \in L^{2}\left(\mathbb{R}^{N} ; \mathbb{C}^{N}\right)$ then by Lemmas 2.11 and $2.12, A u \in L^{2}\left(\mathbb{R}^{N} ; \mathbb{C}^{N}\right)$. Hence $(2.15)$ and $\Longleftarrow$ in (2.14).

- If $(\nabla+\mathrm{i} A) u \in L^{2}\left(\mathbb{R}^{N} ; \mathbb{C}^{N}\right)$ then by Theorem 2.10 and Lemma $2.12, A u \in L^{2}\left(\mathbb{R}^{N} ; \mathbb{C}^{N}\right)$ and $(\nabla+\mathrm{i} A) u \stackrel{\text { a.e. }}{=} \nabla u+\mathrm{i} A u$. Hence, $\nabla u \in L^{2}\left(\mathbb{R}^{N} ; \mathbb{C}^{N}\right)$ and $\Longrightarrow$ in $(2.14)$ is proved.

- By (2.12) and Lemma 2.11, we have

$$
\|\nabla u+\mathrm{i} A u\|_{L^{2}\left(\mathbb{R}^{N}\right)} \leqslant\|\nabla u\|_{L^{2}\left(\mathbb{R}^{N}\right)}+\|A u\|_{L^{2}\left(\mathbb{R}^{N}\right)} \leqslant\left(C \alpha_{A}+1\right)\|u\|_{H^{1}\left(\mathbb{R}^{N}\right)} .
$$

Hence the result.

Lemma 2.14. Let $u, v \in H^{1}\left(\mathbb{R}^{N}\right)$.

1. Let $A \in L_{\text {loc }}^{2}\left(\mathbb{R}^{N} ; \mathbb{R}^{N}\right)$ satisfy (1.3)-(1.4) ((2.1), if $\left.N=1\right)$. Then $(A u) . \nabla v \in L^{1}\left(\mathbb{R}^{N}\right),|A|^{2} u v \in$ $L^{1}\left(\mathbb{R}^{N}\right)$ and we have,

$$
\begin{gathered}
\int_{\mathbb{R}^{N}}|A u||\nabla v| \mathrm{d} x \leqslant C \alpha_{A}\|u\|_{H^{1}\left(\mathbb{R}^{N}\right)}\|v\|_{H^{1}\left(\mathbb{R}^{N}\right)}, \\
\int_{\mathbb{R}^{N}}|A|^{2}|u v| \mathrm{d} x \leqslant C^{2} \alpha_{A}^{2}\|u\|_{H^{1}\left(\mathbb{R}^{N}\right)}\|v\|_{H^{1}\left(\mathbb{R}^{N}\right)} .
\end{gathered}
$$

where the constant $C$ is given by (2.12). 
2. Let $V$ satisfy (1.14) $((2.2)$, if $N=1)$. Then $V u v \in L^{1}\left(\mathbb{R}^{N}\right)$ and we have,

$$
\int_{\mathbb{R}^{N}}|V||u v| \mathrm{d} x \leqslant C \alpha_{V}\|u\|_{H^{1}\left(\mathbb{R}^{N}\right)}\|v\|_{H^{1}\left(\mathbb{R}^{N}\right)}
$$

where the constant $C$ is given by (2.13).

Proof. The results come from Lemma 2.11, Lemma 2.12, and the Cauchy-Schwarz inequality.

From now and until the end of this section, we shall suppose that the assumptions of Theorem 2.3 are fulfilled.

Lemma 2.15. Let us define,

$$
E=\left\{u \in H^{1}\left(\mathbb{R}^{N}\right) ; V u^{2} \in L^{1}\left(\mathbb{R}^{N}\right) \text { and } A u \in L^{2}\left(\mathbb{R}^{N} ; \mathbb{C}^{N}\right)\right\}
$$

Then, $H_{A, V}^{1}\left(\mathbb{R}^{N}\right)=E$ and $\mathscr{D}\left(\mathbb{R}^{N}\right) \hookrightarrow H_{A, V}^{1}\left(\mathbb{R}^{N}\right) \underset{\text { dense }}{\hookrightarrow} H^{1}\left(\mathbb{R}^{N}\right)$. In particular, each term in the integrals of $\langle., .\rangle_{H_{A, V}^{1}\left(\mathbb{R}^{N}\right)}$ belongs to $L^{1}\left(\mathbb{R}^{N}\right)$.

Proof. It is clear that $E \subset H_{A, V}^{1}\left(\mathbb{R}^{N}\right)$. By Lemma 2.13, $H_{A, V}^{1}\left(\mathbb{R}^{N}\right) \subset E \subset H^{1}\left(\mathbb{R}^{N}\right)$. It follows that $H_{A, V}^{1}\left(\mathbb{R}^{N}\right)=E$, which gives the last part of the lemma, with help of Lemma 2.14. Let $u \in H_{A, V}^{1}\left(\mathbb{R}^{N}\right)$. We have by (1.11), (2.12) and (2.11),

$$
\|u\|_{L^{2}\left(\mathbb{R}^{N}\right)}^{2} \leqslant \frac{1}{\nu} \int_{\mathbb{R}^{N}} V|u|^{2} \mathrm{~d} x \leqslant \frac{1}{\nu}\|u\|_{H_{A, V}^{1}}^{2}\left(\mathbb{R}^{N}\right),
$$

and

$$
\begin{aligned}
& \|\nabla u\|_{L^{2}\left(\mathbb{R}^{N}\right)} \leqslant\|\nabla u+\mathrm{i} A u\|_{L^{2}\left(\mathbb{R}^{N}\right)}+\|A u\|_{L^{2}\left(\mathbb{R}^{N}\right)} \\
\leqslant & \|\nabla u+\mathrm{i} A u\|_{L^{2}\left(\mathbb{R}^{N}\right)}+C \alpha_{A}\||u|\|_{H^{1}\left(\mathbb{R}^{N}\right)} \\
\leqslant & \|\nabla u+\mathrm{i} A u\|_{L^{2}\left(\mathbb{R}^{N}\right)}+C \alpha_{A}\left(\|u\|_{L^{2}\left(\mathbb{R}^{N}\right)}+\|\nabla u+\mathrm{i} A u\|_{L^{2}\left(\mathbb{R}^{N}\right)}\right) \\
\leqslant & C\|u\|_{H_{A, V}^{1}\left(\mathbb{R}^{N}\right)} .
\end{aligned}
$$

Hence, $H_{A, V}^{1}\left(\mathbb{R}^{N}\right) \hookrightarrow H^{1}\left(\mathbb{R}^{N}\right)$. Let $\varphi \in \mathscr{D}\left(\mathbb{R}^{N}\right)$. Let $R>0$ be such that $\operatorname{supp} \varphi \subset B(0, R)$. By Hölder's inequality, $V \varphi^{2} \in L^{1}\left(\mathbb{R}^{N}\right)$ and $A \varphi \in L^{2}\left(\mathbb{R}^{N} ; \mathbb{C}^{N}\right)$. It follows that $\varphi \in H_{A, V}^{1}\left(\mathbb{R}^{N}\right)$. Again by Hölder's inequality and (2.16), we have

$$
\|\varphi\|_{H_{A, V}^{1}\left(\mathbb{R}^{N}\right)}^{2} \leqslant\|V\|_{L^{1}(B(0, R))}\|\varphi\|_{L^{\infty}\left(\mathbb{R}^{N}\right)}^{2}+C\|\varphi\|_{H^{1}\left(\mathbb{R}^{N}\right)}^{2},
$$

where $C$ does not depend on $\varphi$. Hence, $\mathscr{D}\left(\mathbb{R}^{N}\right) \hookrightarrow H_{A, V}^{1}\left(\mathbb{R}^{N}\right)$. Finally, since $\mathscr{D}\left(\mathbb{R}^{N}\right) \subset H_{A, V}^{1}\left(\mathbb{R}^{N}\right)$ and $\mathscr{D}\left(\mathbb{R}^{N}\right) \underset{\text { dense }}{\hookrightarrow} H^{1}\left(\mathbb{R}^{N}\right)$, we conclude that $H_{A, V}^{1}\left(\mathbb{R}^{N}\right) \underset{\text { dense }}{\hookrightarrow} H^{1}\left(\mathbb{R}^{N}\right)$. 
Lemma 2.16. It holds that $\mathscr{D}\left(\mathbb{R}^{N}\right) \subset H_{A, V}^{1}\left(\mathbb{R}^{N}\right)$ and for any $u \in H_{A, V}^{1}\left(\mathbb{R}^{N}\right)$, there exists $\left(\varphi_{n}\right)_{n \in \mathbb{N}} \subset$ $\mathscr{D}\left(\mathbb{R}^{N}\right)$ such that $\varphi_{n} \underset{n \rightarrow \infty}{\stackrel{H_{A, V}^{1}}{\longrightarrow}} u$. In addition, we have for any $n \in \mathbb{N},\left\|\varphi_{n}\right\|_{L^{p}\left(\mathbb{R}^{N}\right)} \leqslant\|u\|_{L^{p}\left(\mathbb{R}^{N}\right)}$, for any $p \in[1, \infty]$, as soon as $u \in L^{p}\left(\mathbb{R}^{N}\right)$.

Proof. We adapt the proof of Theorem 7.22, p.194, in Lieb and Loss [8] to handle the presence of the potential $V$ in the integral $\int V|u|^{2} \mathrm{~d} x$. By Lemma 2.16, we already know that $\mathscr{D}\left(\mathbb{R}^{N}\right) \subset H_{A, V}^{1}\left(\mathbb{R}^{N}\right)$. Let $\xi \in C^{\infty}(\mathbb{R} ; \mathbb{R})$ be such that $0 \leqslant \xi \leqslant 1, \xi(t)=1$, if $|t| \leqslant 1$ and $\xi(t)=0$, if $|t| \geqslant 2$. Let $n \in \mathbb{N}$. Set for any $x \in \mathbb{R}^{N}, \xi_{n}(x)=\xi\left(\frac{|x|}{n}\right)$. We denote by $\left(\rho_{n}\right)_{n \in \mathbb{N}} \subset \mathscr{D}\left(\mathbb{R}^{N}\right)$ any standard sequence of mollifiers. Let $u \in H_{A, V}^{1}\left(\mathbb{R}^{N}\right)$. Let $\varepsilon>0$. Let $p \in[1, \infty]$ be such that $u \in L^{p}\left(\mathbb{R}^{N}\right)$. We proceed in three steps. Step 1: There exists $v \in H_{A, V}^{1}\left(\mathbb{R}^{N}\right) \cap L_{\mathrm{c}}^{\infty}\left(\mathbb{R}^{N}\right)$ such that $\|u-v\|_{H_{A, V}^{1}\left(\mathbb{R}^{N}\right)}<\frac{\varepsilon}{2}$ and $|v| \stackrel{\text { a.e. }}{\leqslant}|u|$.

Let $n \in \mathbb{N}$. Let for $x \in \mathbb{R}^{N}, u_{n}(x)=\xi_{n}(x) \xi\left(\frac{|u(x)|}{n}\right) u(x)$. Then, $\operatorname{supp} u_{n} \subset \bar{B}(0,2 n),\left\|u_{n}\right\|_{L^{\infty}\left(\mathbb{R}^{N}\right)} \leqslant 2 n$, $u_{n} \underset{n \rightarrow \infty}{\stackrel{\text { a.e. in } \mathbb{R}^{N}}{\longrightarrow}} u, \sqrt{V} u_{n} \underset{n \rightarrow \infty}{\stackrel{\text { a.e. in } \mathbb{R}^{N}}{\longrightarrow}} \sqrt{V} u,\left|u_{n}\right| \leqslant|u| \in L^{2}\left(\mathbb{R}^{N}\right)$ and $\sqrt{V}\left|u_{n}\right| \leqslant \sqrt{V}|u| \in L^{2}$. It follows that $\left(u_{n}\right)_{n \in \mathbb{N}} \subset L_{\mathrm{c}}^{\infty}\left(\mathbb{R}^{n}\right)$ and by the dominated convergence Theorem,

$$
\lim _{n \rightarrow \infty} \int_{\mathbb{R}^{N}} V\left|u-u_{n}\right|^{2} \mathrm{~d} x=0 \text { and } u_{n} \underset{n \rightarrow \infty}{\stackrel{L^{2}\left(\mathbb{R}^{N}\right)}{\longrightarrow}} u .
$$

In addition,

$$
\nabla u_{n}=\frac{1}{n} \xi^{\prime}\left(\frac{|.|}{n}\right) \xi\left(\frac{|u|}{n}\right) u \frac{x}{|x|}+\frac{1}{n} \xi_{n} \xi^{\prime}\left(\frac{|u|}{n}\right) u \nabla|u|+\xi_{n} \xi\left(\frac{|u|}{n}\right) \nabla u \stackrel{\text { a.e. in } \mathbb{R}^{N}}{\longrightarrow} \nabla u .
$$

But, $\xi^{\prime}\left(\frac{|u|}{n}\right)=0$, if $|u| \geqslant 2 n$ so that,

$$
\left|\nabla u_{n}\right| \leqslant\left\|\xi^{\prime}\right\|_{L^{\infty}(\mathbb{R})}|u|+\left(2\left\|\xi^{\prime}\right\|_{L^{\infty}(\mathbb{R})}+1\right)|\nabla u| \in L^{2}\left(\mathbb{R}^{N}\right),
$$

by Lemmas 2.15 and 2.11. By the dominated convergence Theorem and (2.17), we then infer that $u_{n} \underset{n \rightarrow \infty}{\stackrel{H^{1}\left(\mathbb{R}^{N}\right)}{\longrightarrow}} u$. It follows from $(2.16)-(2.17)$ that $\left(u_{n}\right)_{n \in \mathbb{N}} \subset H_{A, V}^{1}\left(\mathbb{R}^{N}\right)$ and $u_{n} \frac{H_{A, V}^{1}\left(\mathbb{R}^{N}\right)}{n \rightarrow \infty} u$. Pick any $n_{0} \in \mathbb{N}$ large enough to have $\left\|u-u_{n_{0}}\right\|_{H_{A, V}^{1}\left(\mathbb{R}^{N}\right)}<\frac{\varepsilon}{2}$. Hence the result with $v=u_{n_{0}}$.

Step 2: There exists $\varphi \in \mathscr{D}\left(\mathbb{R}^{N}\right)$ such that $\|v-\varphi\|_{H_{A, V}^{1}\left(\mathbb{R}^{N}\right)}<\frac{\varepsilon}{2}$ and $\|\varphi\|_{L^{p}\left(\mathbb{R}^{N}\right)} \leqslant\|v\|_{L^{p}\left(\mathbb{R}^{N}\right)}$.

Let $n \in \mathbb{N}$. Let $R>1$ be such that $B(0, R) \supset \operatorname{supp} v$. Set, $v_{n}=\rho_{n} \star v$. Since $v \in H_{c}^{1}\left(\mathbb{R}^{N}\right)$ it is well-known that $v_{n} \in \mathscr{D}\left(\mathbb{R}^{N}\right),\left\|v_{n}\right\|_{L^{p}\left(\mathbb{R}^{N}\right)} \leqslant\|v\|_{L^{p}\left(\mathbb{R}^{N}\right)}$ (by Young's inequality),

$$
\begin{aligned}
& \operatorname{supp} v_{n} \subset \operatorname{supp} \rho_{n}+\operatorname{supp} v \subset B(0,2 R), \\
& v_{n} \underset{n \rightarrow \infty}{\stackrel{H^{1}\left(\mathbb{R}^{N}\right)}{\longrightarrow}} v
\end{aligned}
$$

(see for instance Brezis [3]: Proposition 4.18, p.106; Proposition 4.20, p.107; Theorem 4.22, p.109; Lemma 9.1, p.266). Then, $v_{n} \in H_{A, V}^{1}\left(\mathbb{R}^{N}\right)$ and by (2.16),

$$
\nabla v_{n}+\mathrm{i} A v_{n} \underset{n \rightarrow \infty}{\stackrel{L^{2}\left(\mathbb{R}^{N}\right)}{\longrightarrow}} \nabla v+\mathrm{i} A v .
$$




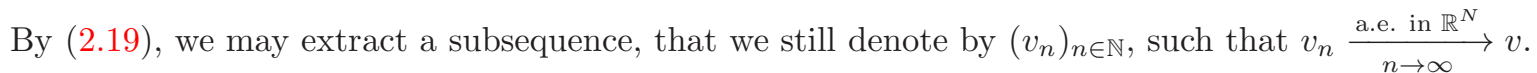
As a consequence, $\sqrt{V} v_{n} \underset{n \rightarrow \infty}{\stackrel{\text { a.e. in } \mathbb{R}^{N}}{\longrightarrow}} \sqrt{V} v$. Applying Young's inequality and (2.18), we see that

$$
\sqrt{V}\left|v_{n}\right| \leqslant \sqrt{V}\|v\|_{L^{\infty}\left(\mathbb{R}^{N}\right)} \mathbb{1}_{B(0,2 R)} \in L^{2}\left(\mathbb{R}^{N}\right) .
$$

It follows from the dominated convergence Theorem that $\lim _{n \rightarrow \infty} \int_{\mathbb{R}^{N}} V\left|v-v_{n}\right|^{2} \mathrm{~d} x=0$, which gives with $(2.20)$,

$$
\lim _{n \rightarrow \infty}\left\|v-v_{n}\right\|_{H_{A, V}^{1}\left(\mathbb{R}^{N}\right)}=0 .
$$

We then choose $\varphi=v_{n_{1}}$, where $n_{1} \in \mathbb{N}$ is sufficiently large to have $\left\|v-v_{n_{1}}\right\|_{H_{A, V}^{1}\left(\mathbb{R}^{N}\right.}<\frac{\varepsilon}{2}$. Hence Step 2.

Step 3: Conclusion.

The result follows from Steps 1 and 2.

Lemma 2.17. The space $H_{A, V}^{1}\left(\mathbb{R}^{N}\right)$ is complete.

Proof. Let $\left(u_{n}\right)_{n \in \mathbb{N}} \subset H_{A, V}^{1}\left(\mathbb{R}^{N}\right)$ be a Cauchy sequence. Since $H_{A, V}^{1}\left(\mathbb{R}^{N}\right) \hookrightarrow H^{1}\left(\mathbb{R}^{N}\right)$ which is

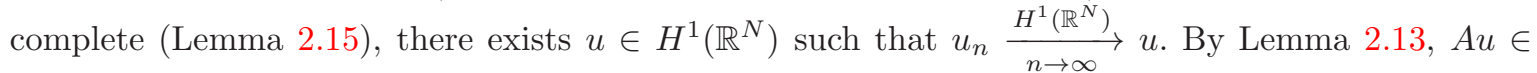
$L^{2}\left(\mathbb{R}^{N} ; \mathbb{C}^{N}\right)$ and $\nabla u_{n}+\mathrm{i} A u_{n} \underset{n \rightarrow \infty}{\stackrel{L^{2}\left(\mathbb{R}^{N}\right)}{\longrightarrow}} \nabla u+\mathrm{i} A u$. To conclude, it remains to show that $\sqrt{V} u \in L^{2}\left(\mathbb{R}^{N}\right)$ and $\sqrt{V} u_{n} \underset{n \rightarrow \infty}{\stackrel{L^{2}\left(\mathbb{R}^{N}\right)}{\longrightarrow}} \sqrt{V} u$. The sequence $\left(\sqrt{V} u_{n}\right)_{n \in \mathbb{N}}$ being Cauchy in $L^{2}\left(\mathbb{R}^{N}\right)$, it is bounded and there exists $v \in L^{2}\left(\mathbb{R}^{N}\right)$ such that $\sqrt{V} u_{n} \frac{L^{2}\left(\mathbb{R}^{N}\right)}{n \rightarrow \infty} v$. There exists a subsequence $\left(u_{n_{k}}\right)_{k \in \mathbb{N}} \subset\left(u_{n}\right)_{n \in \mathbb{N}}$ such

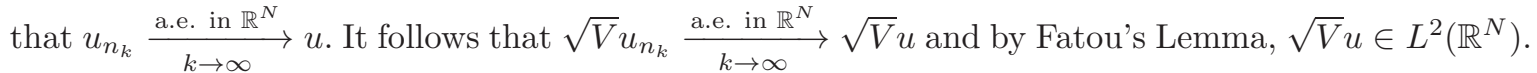
Let $\varphi \in \mathscr{D}\left(\mathbb{R}^{N}\right)$. By Hölder's inequality, $\sqrt{V} \varphi \in L^{2}\left(\mathbb{R}^{N}\right)$. We have for any $n \in \mathbb{N}$,

$$
\left\langle\sqrt{V} u_{n}, \varphi\right\rangle_{L^{2}\left(\mathbb{R}^{N}\right), L^{2}\left(\mathbb{R}^{N}\right)}=\left\langle u_{n}, \sqrt{V} \varphi\right\rangle_{L^{2}\left(\mathbb{R}^{N}\right), L^{2}\left(\mathbb{R}^{N}\right)}
$$

By the above convergences, we can pass to the limit and we get for any $\varphi \in \mathscr{D}\left(\mathbb{R}^{N}\right)$,

$$
\langle v, \varphi\rangle_{L^{2}\left(\mathbb{R}^{N}\right), L^{2}\left(\mathbb{R}^{N}\right)}=\langle u, \sqrt{V} \varphi\rangle_{L^{2}\left(\mathbb{R}^{N}\right), L^{2}\left(\mathbb{R}^{N}\right)}=\langle\sqrt{V} u, \varphi\rangle_{L^{2}\left(\mathbb{R}^{N}\right), L^{2}\left(\mathbb{R}^{N}\right)} .
$$

It follows that, $v=\sqrt{V} u$ in $\mathscr{D}^{\prime}\left(\mathbb{R}^{N}\right)$ and so in $L^{2}\left(\mathbb{R}^{N}\right)$. The lemma is proved.

Proof of Theorem 2.5. By Lemma 2.15, $H_{A, V}^{1}\left(\mathbb{R}^{N}\right) \hookrightarrow H^{1}\left(\mathbb{R}^{N}\right)$. It remains to show that, $H^{1}\left(\mathbb{R}^{N}\right) \hookrightarrow H_{A, V}^{1}\left(\mathbb{R}^{N}\right)$. Let $u \in H^{1}\left(\mathbb{R}^{N}\right)$. Then, $|u| \in H^{1}\left(\mathbb{R}^{N}\right)$ and $\||u|\|_{H^{1}\left(\mathbb{R}^{N}\right)} \leqslant\|u\|_{H^{1}\left(\mathbb{R}^{N}\right)}$ (Lemma 2.11). By Lemma 2.12, $A u \in L^{2}\left(\mathbb{R}^{N} ; \mathbb{C}^{N}\right)$ and $V u^{2} \in L^{1}\left(\mathbb{R}^{N}\right)$. As a consequence, $u \in$ $H_{A, V}^{1}\left(\mathbb{R}^{N}\right)$ and by (2.13) and (2.16),

$$
\|u\|_{H_{A, V}^{1}\left(\mathbb{R}^{N}\right)} \leqslant C\|u\|_{H^{1}\left(\mathbb{R}^{N}\right)}
$$


where $C$ does not depend on $u$.

Lemma 2.18. The space $H_{A, V}^{1}\left(\mathbb{R}^{N}\right)$ is separable.

Proof. By Theorem 2.5, $H_{A, 1}^{1}\left(\mathbb{R}^{N}\right)=H^{1}\left(\mathbb{R}^{N}\right)$ with equivalent norms. Let $H=H^{1}\left(\mathbb{R}^{N}\right)$, with $\|\cdot\|_{H}=\|\cdot\|_{H_{A, 1}^{1}\left(\mathbb{R}^{N}\right)}$. Since $\left(H^{1}\left(\mathbb{R}^{N}\right),\|\cdot\|_{H^{1}\left(\mathbb{R}^{N}\right)}\right)$ is separable, so is $\left(H,\|\cdot\|_{H}\right)$. Let us define the linear operator $T$ by,

$$
\begin{aligned}
T: H_{A, V}^{1}\left(\mathbb{R}^{N}\right) & \longrightarrow L^{2}\left(\mathbb{R}^{N}\right) \times H \\
u & \longmapsto(\sqrt{V} u, u),
\end{aligned}
$$

with $\|(u, v)\|_{L^{2}\left(\mathbb{R}^{N}\right) \times H}^{2}=\|u\|_{L^{2}\left(\mathbb{R}^{N}\right)}^{2}+\|v\|_{H}^{2}$, for any $(u, v) \in L^{2}\left(\mathbb{R}^{N}\right) \times H$. Clearly, $L^{2}\left(\mathbb{R}^{N}\right) \times H$ is separable. Thus, $T\left(H_{A, V}^{1}\left(\mathbb{R}^{N}\right)\right)$ is also separable (Brezis [3]: Proposition 3.25, p.73). But for any $u \in H_{A, V}^{1}\left(\mathbb{R}^{N}\right)$,

$$
\|T(u)\|_{L^{2}\left(\mathbb{R}^{N}\right) \times H} \geqslant\|u\|_{H_{A, V}^{1}\left(\mathbb{R}^{N}\right)},
$$

so that $H_{A, V}^{1}\left(\mathbb{R}^{N}\right)$ is separable.

Proof of Theorem 2.3. By Lemmas 2.15-2.18, it remains to show the continuous embeddings and the densities in (2.7). This comes from the fact that the embeddings in (2.6) are dense and from the reflexivity of the spaces $\mathscr{D}\left(\mathbb{R}^{N}\right)$ and $H_{A, V}^{1}\left(\mathbb{R}^{N}\right)$.

Proof of Theorem 2.7. Estimate (2.8) comes from (1.15)-(1.17) and a straightforward calculation, while (2.9) is a consequence of the embeddings $H_{A, V}^{1}\left(\mathbb{R}^{N}\right) \hookrightarrow H^{1}\left(\mathbb{R}^{N}\right)$ and $H^{-1}\left(\mathbb{R}^{N}\right) \hookrightarrow H_{A, V}^{-1}\left(\mathbb{R}^{N}\right)$, due to (2.6) and (2.7).

\section{The set of dislocations}

Lemma 3.1. Let $A$ satisfy (1.3), (1.5) and (1.6). Then for any $y \in \mathbb{Z}^{N}$, there exists a unique continuous function $\psi_{y} \in W_{\mathrm{loc}}^{1, N+\varepsilon}\left(\mathbb{R}^{N} ; \mathbb{R}\right)\left(\psi_{y} \in H_{\mathrm{loc}}^{1}(\mathbb{R} ; \mathbb{R})\right.$, if $\left.N=1\right)$ such that

$$
\begin{gathered}
\psi_{y}(0)=0 \\
\forall x \in \mathbb{R}^{N}, \psi_{y}(x-y)+\psi_{-y}(x)=\psi_{y}(-y)=\psi_{-y}(y), \\
A(x+y)=A(x)+\nabla \psi_{y}(x),
\end{gathered}
$$

for almost every $x \in \mathbb{R}^{N}$. In particular, $\psi_{0}=0$ over $\mathbb{R}^{N}$. 
Proof. Let $y \in \mathbb{Z}^{N}$. Uniqueness for $\psi_{y}$ comes from (3.1) and (3.3), once continuity is proved. By Remark 1.2 and the Sobolev embedding, there exists $\widetilde{\psi_{y}} \in W_{\text {loc }}^{1, N+\varepsilon}\left(\mathbb{R}^{N} ; \mathbb{R}\right)$ satisfying (3.3) and continuous over $\mathbb{R}^{N}$. Setting $\psi_{y}=\widetilde{\psi_{y}}-\widetilde{\psi_{y}}(0)$, we see that $\psi_{y}$ verifies (3.1) and (3.3). Notice that the function $x \longmapsto 0$ satisfies (3.3) for $y=0$, so that $\psi_{0}=0$, by uniqueness. It remains to establish (3.2). Applying (3.3) with $y$ at the point $x-y$ and a second time with $-y$, we obtain for almost every $x \in \mathbb{R}^{N}$,

$$
A(x-y)=A(x)-\nabla \psi_{y}(x-y)=A(x)+\nabla \psi_{-y}(x)
$$

It follows that there exists $c \in \mathbb{R}$ such that,

$$
\forall x \in \mathbb{R}^{N}, \psi_{y}(x-y)+\psi_{-y}(x)=c .
$$

Substituting first $x=0$, then $x=y$ and using (3.1) we obtain (3.2).

Lemma 3.2. Let A satisfy (1.3), (1.5) and (1.6). Let $\left(\psi_{y}\right)_{y \in \mathbb{Z}^{N}}$ be given by Lemma 3.1. For any $y \in \mathbb{Z}^{N}$, let $\varphi_{y} \in W_{\mathrm{loc}}^{1, N+\varepsilon}\left(\mathbb{R}^{N} ; \mathbb{R}\right)$ be defined by,

$$
\varphi_{y} \stackrel{\text { def }}{=} \psi_{y}-\frac{1}{2} \psi_{y}(-y)
$$

Then $\varphi_{y} \in C\left(\mathbb{R}^{N} ; \mathbb{R}\right)$ and verifies,

$$
\begin{gathered}
\forall x \in \mathbb{R}^{N}, \varphi_{y}(x-y)+\varphi_{-y}(x)=0, \\
A(x+y)=A(x)+\nabla \varphi_{y}(x),
\end{gathered}
$$

for almost every $x \in \mathbb{R}^{N}$. Finally, $\varphi_{0}=0$ over $\mathbb{R}^{N}$.

Proof. By Lemma 3.1 and (3.4), we only have to check (3.5). The result then comes from (3.4) and $(3.2)$.

Assume that $A$ and $V$ satisfy Assumptions 1.1 and 1.3, respectively. For any $y \in \mathbb{Z}^{N}$, we define $\tau_{y} \in \mathscr{L}\left(H_{A, V}^{1}\left(\mathbb{R}^{N}\right)\right)$ as follows.

$$
\begin{aligned}
\tau_{y}: H_{A, V}^{1}\left(\mathbb{R}^{N}\right) & \longrightarrow H_{A, V}^{1}\left(\mathbb{R}^{N}\right) \\
u & \longmapsto e^{\mathrm{i} \varphi_{y}} u(\cdot+y),
\end{aligned}
$$

where $\varphi_{y}$ is given by (3.4). Indeed, it is clear that $\tau_{y}: H_{A, V}^{1}\left(\mathbb{R}^{N}\right) \longrightarrow L^{2}\left(\mathbb{R}^{N}\right)$ is linear and,

$$
\int_{\mathbb{R}^{N}} V\left|\tau_{y} u\right|^{2} \mathrm{~d} x=\int_{\mathbb{R}^{N}} V|u|^{2} \mathrm{~d} x,
$$


for any $u \in H_{A, V}^{1}\left(\mathbb{R}^{N}\right)$. In addition, by (3.6), we have for any $y \in \mathbb{Z}^{N}, u \in H_{A, V}^{1}\left(\mathbb{R}^{N}\right)$ and almost every $x \in \mathbb{R}^{N}$,

$$
\begin{aligned}
& \nabla\left(\tau_{y} u\right)(x)+\mathrm{i} A(x)\left(\tau_{y} u\right)(x) \\
= & \left(\nabla u(x+y)+\mathrm{i} A(x) u(x+y)+\mathrm{i} \nabla \varphi_{y}(x) u(x+y)\right) e^{\mathrm{i} \varphi_{y}(x)} \\
= & (\nabla u(x+y)+\mathrm{i} A(x+y) u(x+y)) e^{\mathrm{i} \varphi_{y}(x)}
\end{aligned}
$$

We deduce that $\tau_{y}: H_{A, V}^{1}\left(\mathbb{R}^{N}\right) \longrightarrow H_{A, V}^{1}\left(\mathbb{R}^{N}\right)$ is well-defined, linear and

$$
\left\|\nabla\left(\tau_{y} u\right)+\mathrm{i} A\left(\tau_{y} u\right)\right\|_{L^{2}\left(\mathbb{R}^{N}\right)}=\|\nabla u+\mathrm{i} A u\|_{L^{2}\left(\mathbb{R}^{N}\right)} .
$$

The above estimates and (3.7) permit us to see that for any $y \in \mathbb{Z}^{N}, \tau_{y} \in \mathscr{L}\left(H_{A, V}^{1}\left(\mathbb{R}^{N}\right)\right)$ with $\left\|\tau_{y}\right\|_{\mathscr{L}\left(H_{A, V}^{1}\left(\mathbb{R}^{N}\right)\right)}=1$. Let

$$
D \stackrel{\text { def }}{=}\left\{\tau_{y} ; y \in \mathbb{Z}^{N}\right\}
$$

Proposition 3.3. Let $A$ and $V$ satisfy Assumptions 1.1 and 1.3 , respectively, and let $D$ be defined by (3.8). Then $D$ is a set of unitary operators on $H_{A, V}^{1}\left(\mathbb{R}^{N}\right)$. In addition,

$$
\begin{gathered}
\tau_{0}=\mathrm{Id}, \\
\tau_{y}^{-1}=\tau_{y}^{\star}=\tau_{-y}, \\
\left\langle\tau_{y} u, \tau_{y} v\right\rangle_{H_{A, V}^{1}\left(\mathbb{R}^{N}\right)}=\langle u, v\rangle_{H_{A, V}^{1}\left(\mathbb{R}^{N}\right)},
\end{gathered}
$$

for any $y \in \mathbb{Z}^{N}$ and $u, v \in H_{A, V}^{1}\left(\mathbb{R}^{N}\right)$.

Proof of Lemma 3.3. Recall that $D$ is set of bounded linear operators on $H_{A, V}^{1}\left(\mathbb{R}^{N}\right)$. By Lemma 3.2, $\varphi_{0}=0$ so that $\tau_{0}=\mathrm{Id}$. Let $y \in \mathbb{Z}^{N}$ and let $u \in H_{A, V}^{1}\left(\mathbb{R}^{N}\right)$. For almost every $x \in \mathbb{R}^{N}$, one has,

$$
\tau_{y}\left(\tau_{-y} u\right)(x)=e^{\mathrm{i} \varphi_{y}(x)}\left(\tau_{-y} u\right)(x+y)=e^{\mathrm{i} \varphi_{y}(x)} e^{\mathrm{i} \varphi_{-y}(x+y)} u(x)=u(x),
$$

where we have used (3.5) in the last equality. Still with (3.5), we show that $\tau_{-y}\left(\tau_{y} u\right)=u$. It follows that $\tau_{y}$ is invertible and $\tau_{y}^{-1}=\tau_{-y}$. Now, let $v \in H_{A, V}^{1}\left(\mathbb{R}^{N}\right)$. By a straightforward calculation and with help of (3.5) again and (3.6), we obtain

$$
\left\langle u, \tau_{y}^{\star} v\right\rangle_{H_{A, V}^{1}\left(\mathbb{R}^{N}\right)} \stackrel{\text { def }}{=}\left\langle\tau_{y} u, v\right\rangle_{H_{A, V}^{1}\left(\mathbb{R}^{N}\right)}=\left\langle u, \tau_{y}^{-1} v\right\rangle_{H_{A, V}^{1}\left(\mathbb{R}^{N}\right)},
$$

so that, $\tau_{y}^{\star}=\tau_{y}^{-1}$ which concludes the proof.

Let us recall the following definition (see Definition 3.1, p.60, in Tintarev and Fieseler [14] and Proposition 3.3). 
Definition 3.4. Let $A$ and $V$ satisfy Assumptions 1.1 and 1.3, respectively, and let $D$ be defined by (3.8). Let $\left(u_{n}\right)_{n \in \mathbb{N}} \subset H_{A, V}^{1}\left(\mathbb{R}^{N}\right)$ and $u \in H_{A, V}^{1}\left(\mathbb{R}^{N}\right)$. We shall say that the sequence $\left(u_{n}\right)_{n \in \mathbb{N}}$ converges to $u D$-weakly, which we will denote as,

$$
u_{n} \underset{n \rightarrow \infty}{\stackrel{D}{\longrightarrow}} u,
$$

if

$$
\lim _{n \rightarrow \infty}\left\langle u_{n}-u, \tau_{y_{n}} v\right\rangle_{H_{A, V}^{1}\left(\mathbb{R}^{N}\right)}=0,
$$

for any sequence $\left(\tau_{y_{n}}\right)_{n \in \mathbb{N}} \subset D$ and $v \in H_{A, V}^{1}\left(\mathbb{R}^{N}\right)$.

Notation 3.5. Let $A$ and $V$ satisfy Assumptions 1.1 and 1.3, respectively, and let $D$ be defined by (3.8). Let $\left(\tau_{y_{n}}\right)_{n \in \mathbb{N}} \subset D$. We shall write,

$$
\tau_{y_{n}} \underset{n \rightarrow \infty}{\longrightarrow} 0
$$

to mean that for any $u \in H_{A, V}^{1}\left(\mathbb{R}^{N}\right), \tau_{y_{n}} u \underset{n \rightarrow \infty}{\longrightarrow} 0$ in $H_{A, V}^{1}$-weakly, or equivalently,

$$
\lim _{n \rightarrow \infty}\left\langle\tau_{y_{n}} u, v\right\rangle_{H_{A, V}^{1}\left(\mathbb{R}^{N}\right)}=0,
$$

for any $u, v \in H_{A, V}^{1}\left(\mathbb{R}^{N}\right)$.

Remark 3.6. If $u_{n} \underset{n \rightarrow \infty}{D} 0$ then $u_{n} \underset{n \rightarrow \infty}{\longrightarrow} 0$ in $H_{A, V^{-}}^{1}$ weakly. In particular, $u_{n} \underset{n \rightarrow \infty}{\stackrel{H_{w}^{1}}{\longrightarrow}} 0$ and for any sequence $\left(\tau_{y_{n}}\right)_{n \in \mathbb{N}} \subset D, \tau_{y_{n}} u_{n} \underset{n \rightarrow \infty}{\stackrel{H_{w}^{1}}{\longrightarrow}} 0$. Indeed, this follows from (3.9), (3.10) and (2.6).

Lemma 3.7. Let $A$ and $V$ satisfy Assumptions 1.1 and 1.3 , respectively. Let $\left(y_{k}\right)_{k},\left(z_{k}\right)_{k} \subset \mathbb{Z}^{N}$. Then,

$$
\tau_{y_{k}} \tau_{z_{k}} \underset{k \rightarrow \infty}{\longrightarrow} 0 \Longleftrightarrow\left|y_{k}+z_{k}\right| \underset{k \rightarrow \infty}{\longrightarrow} \infty .
$$

Moreover, if $\tau_{y_{k}} \tau_{z_{k}} \underset{k \rightarrow \infty}{\not} 0$ then $\left(\tau_{y_{k}} \tau_{z_{k}}\right)_{k}$ admits a constant subsequence.

Proof. Let $\left(y_{k}\right)_{k},\left(z_{k}\right)_{k} \subset \mathbb{Z}^{N}$.

Step 1: If $\liminf _{k \rightarrow \infty}\left|y_{k}+z_{k}\right|<\infty$ then $\left(y_{k}+z_{k}\right)_{k}$ admits a constant subsequence.

Indeed, if $\liminf _{k \rightarrow \infty}\left|y_{k}+z_{k}\right|<\infty$ then $\left(y_{k}+z_{k}\right)_{k}$ admits a bounded subsequence, from which we extract a convergent subsequence $\left(y_{k_{\ell}}+z_{k_{\ell}}\right)_{\ell}$. Since $\left(y_{k_{\ell}}+z_{k_{\ell}}\right)_{\ell}$ converges in $\mathbb{Z}^{N}$, Step 1 follows.

Step 2: Proof of $\Longrightarrow$.

We show the contraposition. Assume that $\liminf _{k \rightarrow \infty}\left|y_{k}+z_{k}\right|<\infty$. By Step 1, there exists $\left(y_{k_{\ell}}+z_{k_{\ell}}\right)_{\ell} \subset$ 
$\left(y_{k}+z_{k}\right)_{k}$ such that for any $\ell \in \mathbb{N}, y_{k_{\ell}}+z_{k_{\ell}}=y_{k_{1}}+z_{k_{1}}$. Let $u \in H_{A, V}^{1}\left(\mathbb{R}^{N}\right) \backslash\{0\}$ and $v=\tau_{y_{k_{1}}} \tau_{z_{k_{1}}} u$. It follows from (3.11) that,

$$
\forall \ell \in \mathbb{N},\left\langle\tau_{y_{k_{\ell}}} \tau_{z_{k_{\ell}}} u, v\right\rangle_{H_{A, V}^{1}\left(\mathbb{R}^{N}\right)}=\|u\|_{H_{A, V}^{1}\left(\mathbb{R}^{N}\right)}^{2}>0,
$$

and so, $\tau_{y_{k}} \underset{k \rightarrow \infty}{\not} 0$.

Step 3: Proof of $\Longleftarrow$.

Assume $\left|y_{k}+z_{k}\right| \underset{k \rightarrow \infty}{\longrightarrow} \infty$. Let $\varphi, \psi \in \mathscr{D}\left(\mathbb{R}^{N}\right)$. Then for any $k \in \mathbb{N}$ large enough, $\operatorname{supp}\left(\tau_{y_{k}} \tau_{z_{k}} \varphi\right) \cap$ $\operatorname{supp} \psi=\emptyset$, so that,

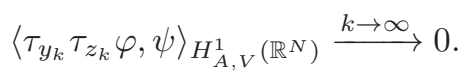

Let $u, v \in H_{A, V}^{1}\left(\mathbb{R}^{N}\right)$. Let $\varepsilon>0$. By density (Theorem 2.3), there exists $\left(\varphi_{n}\right)_{n},\left(\psi_{n}\right)_{n} \subset \mathscr{D}\left(\mathbb{R}^{N}\right)$ such that, $\varphi_{n} \underset{n \rightarrow \infty}{\stackrel{H_{A, V}^{1}\left(\mathbb{R}^{N}\right)}{\longrightarrow}} u$ and $\psi_{n} \underset{n \rightarrow \infty}{\stackrel{H_{A, V}^{1}\left(\mathbb{R}^{N}\right)}{\longrightarrow}} v$. Let $n_{0} \in \mathbb{N}$ be such that,

$$
\|v\|_{H_{A, V}^{1}\left(\mathbb{R}^{N}\right)}\left\|u-\varphi_{n_{0}}\right\|_{H_{A, V}^{1}\left(\mathbb{R}^{N}\right)}+\left\|\varphi_{n_{0}}\right\|_{H_{A, V}^{1}\left(\mathbb{R}^{N}\right)}\left\|v-\psi_{n_{0}}\right\|_{H_{A, V}^{1}\left(\mathbb{R}^{N}\right)} \leqslant \varepsilon
$$

for any $n \geqslant n_{0}$. We then infer with help of (3.11) and Cauchy-Schwarz's inequality that for any $k \in \mathbb{N}$,

$$
\begin{aligned}
& \left|\left\langle\tau_{y_{k}} \tau_{z_{k}} u, v\right\rangle_{H_{A, V}^{1}}\right| \leqslant\left|\left\langle\tau_{y_{k}} \tau_{z_{k}}\left(u-\varphi_{n_{0}}\right), v\right\rangle_{H_{A, V}^{1}}\right|+\left|\left\langle\tau_{y_{k}} \tau_{z_{k}} \varphi_{n_{0}}, v-\psi_{n_{0}}\right\rangle_{H_{A, V}^{1}}\right|+\left|\left\langle\tau_{y_{k}} \tau_{z_{k}} \varphi_{n_{0}}, \psi_{n_{0}}\right\rangle_{H_{A, V}^{1}}\right| \\
\leqslant & \|v\|_{H_{A}^{1}}\left\|u-\varphi_{n_{0}}\right\|_{H_{A, V}^{1}}+\left\|\varphi_{n_{0}}\right\|_{H_{A}^{1}}\left\|v-\psi_{n_{0}}\right\|_{H_{A, V}^{1}}+\left|\left\langle\tau_{y_{k}} \tau_{z_{k}} \varphi_{n_{0}}, \psi_{n_{0}}\right\rangle_{H_{A, V}^{1}}\right| \\
\leqslant & \varepsilon+\left|\left\langle\tau_{y_{k}} \tau_{z_{k}} \varphi_{n_{0}}, \psi_{n_{0}}\right\rangle_{H_{A, V}^{1}}\right| .
\end{aligned}
$$

By (3.13), if follows that: $\limsup _{k \rightarrow \infty}\left|\left\langle\tau_{y_{k}} \tau_{z_{k}} u, v\right\rangle_{H_{A, V}^{1}\left(\mathbb{R}^{N}\right)}\right| \leqslant \varepsilon$. Since $\varepsilon>0$ is arbitrary, we then get that for any $u, v \in H_{A, V}^{1}\left(\mathbb{R}^{N}\right),\left\langle\tau_{y_{k}} \tau_{z_{k}} u, v\right\rangle_{H_{A, V}^{1}\left(\mathbb{R}^{N}\right)} \stackrel{k \rightarrow \infty}{\longrightarrow} 0$, which is the desired result.

Step 4: If $\tau_{y_{k}} \tau_{z_{k}} \underset{k \rightarrow \infty}{\not} 0$ then $\left(\tau_{y_{k}} \tau_{z_{k}}\right)_{k}$ admits a constant subsequence.

Now assume that $\tau_{y_{k}} \tau_{z_{k}} \underset{k \rightarrow \infty}{\not}$. By (3.12), this means $\liminf _{k \rightarrow \infty}\left|y_{k}+z_{k}\right|<\infty$, and we conclude with help of Step 1.

Proposition 3.8. Let $A$ and $V$ satisfy Assumptions 1.1 and 1.3, respectively, and let $D$ be defined by (3.8). Then $D$ is a set of dislocations on $H_{A, V}^{1}\left(\mathbb{R}^{N}\right)$.

Proof. By Proposition 3.1 p.61 in Tintarev and Fieseler [14], it is sufficient to show that if $\left(y_{k}\right)_{k} \subset \mathbb{Z}^{N}$ is such that $\tau_{y_{k}} \underset{k \rightarrow \infty}{<0}$ then $\tau_{y_{k}}$ has a strongly convergence subsequence. This is a consequence of (3.9) and Lemma 3.7. 


\section{Cocompactness}

Theorem 4.1. Let $A$ and $V$ satisfy Assumptions 1.1 and 1.3, respectively, and let $D$ be defined by (3.8). Let $\left(u_{k}\right)_{k \in \mathbb{N}}$ be a bounded sequence in $H_{A, V}^{1}\left(\mathbb{R}^{N}\right)$. Let $p \in\left(2,2^{\star}\right)(p \in(2, \infty]$, if $N=1)$. Then $\left(u_{k}\right)_{k \in \mathbb{N}}$ is bounded in $H^{1}\left(\mathbb{R}^{N}\right)$ and we have the following result.

$$
u_{k} \underset{k \rightarrow \infty}{\stackrel{D}{\longrightarrow}} 0 \Longleftrightarrow u_{k} \underset{k \rightarrow \infty}{\stackrel{L^{p}\left(\mathbb{R}^{N}\right)}{\longrightarrow} 0 .}
$$

Proof. Let $\left(u_{k}\right)_{k \in \mathbb{N}}$ be a bounded sequence in $H_{A, V}^{1}\left(\mathbb{R}^{N}\right)$ and let $p$ be as in the theorem. By (2.6) and Sobolev' embedding, $\left(u_{k}\right)_{k \in \mathbb{N}}$ is bounded in $H^{1}\left(\mathbb{R}^{N}\right)$ and so in $L^{p}\left(\mathbb{R}^{N}\right)$. Assume that $u_{k} \underset{k \rightarrow \infty}{D} 0$. By Remark 3.6, $\tau_{y_{k}} u_{k} \underset{k \rightarrow \infty}{\stackrel{H_{\mathrm{w}}^{1}}{\longrightarrow}} 0$, for any $\left(\tau_{y_{k}}\right)_{k} \subset D$. Suppose $p<\infty$. We claim that,

$$
\forall k \in \mathbb{N}, \exists y_{k} \in \mathbb{Z}^{N} \text { such that } \sup _{y \in \mathbb{Z}^{N}} \int_{Q-y}\left|u_{k}\right|^{p} \mathrm{~d} x=\int_{Q}\left|\tau_{y_{k}} u_{k}\right|^{p} \mathrm{~d} x .
$$

Indeed, if $\sup _{y \in \mathbb{Z}^{N}} \int_{Q-y}\left|u_{k}\right|^{p} \mathrm{~d} x=0$, there is nothing to prove. If $\sup _{y \in \mathbb{Z}^{N}} \int_{Q-y}\left|u_{k}\right|^{p} \mathrm{~d} x=\delta>0$ then if the supremum in $y$ was not a maximum then there would be an infinite number of $y \in \mathbb{Z}^{N}$ such that $\int_{Q-y}\left|u_{k}\right|^{p} \mathrm{~d} x>\frac{\delta}{2}$, contradicting the fact that $\left(u_{k}\right)_{k}$ is bounded in $L^{p}\left(\mathbb{R}^{N}\right)$.

By the Sobolev embedding $H^{1}(Q) \hookrightarrow L^{p}(Q)$ and translation, there exists $C>0$ such that for any $k \in \mathbb{N}$ and $y \in \mathbb{Z}^{N},\left\|u_{k}\right\|_{L^{p}(Q-y)}^{2} \leqslant C\left\|u_{k}\right\|_{H^{1}(Q-y)}^{2}$. Multiplying the both sides by $\|u\|_{L^{p}(Q-y)}^{p-2}$, we get

$$
\int_{Q-y}\left|u_{k}\right|^{p} \mathrm{~d} x \leqslant C\left\|u_{k}\right\|_{H^{1}(Q-y)}^{2}\left(\int_{Q-y}\left|u_{k}\right|^{p} \mathrm{~d} x\right)^{\frac{p-2}{p}} .
$$

Summing over $y \in \mathbb{Z}^{N}$, we obtain for any $k \in \mathbb{N}$,

$$
\left\|u_{k}\right\|_{L^{p}\left(\mathbb{R}^{N}\right)}^{p} \leqslant C\left\|u_{k}\right\|_{H^{1}\left(\mathbb{R}^{N}\right)}^{2} \sup _{y \in \mathbb{Z}^{N}}\left(\int_{Q-y}\left|u_{k}\right|^{p} \mathrm{~d} x\right)^{\frac{p-2}{p}} .
$$

For any $k \in \mathbb{N}$, let $y_{k} \in \mathbb{Z}^{N}$ be given by (4.1). Noticing that $\sup _{k \in \mathbb{N}}\left\|u_{k}\right\|_{H^{1}\left(\mathbb{R}^{N}\right)}<\infty$, we infer from the compactness of the Sobolev embedding $H^{1}(Q) \hookrightarrow L^{p}(Q)$ that

$$
\forall k \in \mathbb{N},\left\|u_{k}\right\|_{L^{p}\left(\mathbb{R}^{N}\right)}^{p} \leqslant C\left\|\tau_{y_{k}} u_{k}\right\|_{L^{p}(Q)}^{p-2} \stackrel{k \rightarrow \infty}{\longrightarrow} 0,
$$

since $\tau_{y_{k}} u_{k} \underset{k \rightarrow \infty}{\stackrel{H_{\mathrm{w}}^{1}}{\longrightarrow}} 0$. When $N=1$ and $p=\infty$, we use the above result and Gagliardo-Nirenberg's inequality to see that,

$$
\left\|u_{k}\right\|_{L^{\infty}(\mathbb{R})} \leqslant C\left\|u_{k}\right\|_{L^{4}(\mathbb{R})}^{\frac{2}{3}}\left\|u_{k}\right\|_{H^{1}(\mathbb{R})}^{\frac{1}{3}} \leqslant C\left\|u_{k}\right\|_{L^{4}(\mathbb{R})}^{\frac{2}{3}} \stackrel{k \rightarrow \infty}{\longrightarrow} 0 .
$$




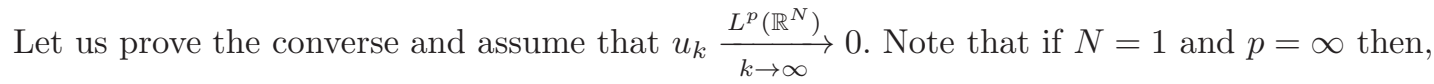

$$
\left\|u_{k}\right\|_{L^{4}(\mathbb{R})}^{2} \leqslant\left\|u_{k}\right\|_{L^{2}(\mathbb{R})}\left\|u_{k}\right\|_{L^{\infty}(\mathbb{R})} \leqslant C\left\|u_{k}\right\|_{L^{\infty}(\mathbb{R})} \stackrel{k \rightarrow \infty}{\longrightarrow} 0 .
$$

So we may assume that $p<\infty$. Let $\left(\tau_{y_{k}}\right)_{k} \in D$. Since for any $k \in \mathbb{N},\left\|\tau_{y_{k}} u_{k}\right\|_{L^{p}\left(\mathbb{R}^{N}\right)}=\left\|u_{k}\right\|_{L^{p}\left(\mathbb{R}^{N}\right)}$ and $\left\|\tau_{y_{k}} u_{k}\right\|_{H_{A, V}^{1}\left(\mathbb{R}^{N}\right)}=\left\|u_{k}\right\|_{H_{A, V}^{1}\left(\mathbb{R}^{N}\right)}$ by $(3.11)$, we obtain that for some $\left(\tau_{y_{k_{\ell}}}\right)_{\ell} \subset\left(\tau_{y_{k}}\right)_{k}$ and $u \in$ $H_{A, V}^{1}\left(\mathbb{R}^{N}\right)$,

$$
\begin{gathered}
\tau_{y_{k}} u_{k} \underset{k \rightarrow \infty}{\longrightarrow} 0, \text { in } L^{p}\left(\mathbb{R}^{N}\right), \\
\tau_{y_{k_{\ell}}} u_{k_{\ell}} \underset{\ell \rightarrow \infty}{\longrightarrow} u \text {, in } H_{A, V^{-}}^{1} \text {-weakly. }
\end{gathered}
$$

In particular, both convergences hold in $\mathscr{D}^{\prime}\left(\mathbb{R}^{N}\right)$, so that $u=0$ and $\tau_{y_{k}} u_{k} \underset{k \rightarrow \infty}{\longrightarrow} 0$, in $H_{A, V^{-}}^{1}$-weakly, for the whole sequence $\left(\tau_{y_{k}} u_{k}\right)_{k}$. This concludes the proof.

\section{An associated critical value function and proof of the main result}

Proposition 5.1. Let $N \geqslant 1$. Let $g$ and $F$ be as in Assumption 1.3, where $f$ satisfies (1.8) and (1.9) and let $\psi$ be defined by (1.7). Then the following holds.

1. $\psi \in C^{1}\left(H^{1}\left(\mathbb{R}^{N}\right) ; \mathbb{R}\right), \psi^{\prime}=g$ and $\psi$ and $\psi^{\prime}$ are bounded on bounded sets.

2. $\forall(u, v) \in H^{1}\left(\mathbb{R}^{N}\right) \times H^{1}\left(\mathbb{R}^{N}\right),\langle g(u), v\rangle_{H^{-1}\left(\mathbb{R}^{N}\right), H^{1}\left(\mathbb{R}^{N}\right)}=\operatorname{Re} \int_{\mathbb{R}^{N}} g(u)(x) \overline{v(x)} \mathrm{d} x$.

3. Let $\left(u_{n}\right)_{n},\left(v_{n}\right)_{n} \subset H^{1}\left(\mathbb{R}^{N}\right)$ be bounded. If $\lim _{n \rightarrow \infty}\left\|u_{n}-v_{n}\right\|_{L^{p}\left(\mathbb{R}^{N}\right)}=0$, for some $p \in[1, \infty]$, then $\lim _{n \rightarrow \infty}\left|\psi\left(u_{n}\right)-\psi\left(v_{n}\right)\right|=0$.

4. Let $u \in H^{1}\left(\mathbb{R}^{N}\right)$. If $u_{n} \frac{H_{w}^{1}}{n \rightarrow \infty} u$ then $g\left(u_{n}\right) \frac{H_{w}^{-1}}{n \rightarrow \infty} g(u)$.

Proposition 5.1 is well-known but with some slightly different assumptions on $f$ and, in all cases, for real-valued functions. It can be adapted and for the convenience of the reader, we postpone its proof to the Appendix A.

Remark 5.2. If $N \geqslant 3$ then, under the hypotheses of Proposition 5.1, the conclusions may be slightly more general as follows. We first recall that,

$$
\begin{gathered}
\mathscr{D}\left(\mathbb{R}^{N}\right) \hookrightarrow E \stackrel{\text { def }}{=} L^{2}\left(\mathbb{R}^{N}\right) \cap L^{2^{\star}}\left(\mathbb{R}^{N}\right) \text { with dense embedding, } \\
E^{\star}=L^{2}\left(\mathbb{R}^{N}\right)+L^{2^{\star \prime}}\left(\mathbb{R}^{N}\right) \hookrightarrow \mathscr{D}^{\prime}\left(\mathbb{R}^{N}\right) \text { with dense embedding, }
\end{gathered}
$$


where, $\|u\|_{E}=\|u\|_{L^{2}\left(\mathbb{R}^{N}\right)}+\|u\|_{L^{2^{\star}\left(\mathbb{R}^{N}\right)}}$. See, for instance, Bergh and Löfström [2] (Lemma 2.3.1, p.24-25 and Theorem 2.7.1, p.32). Then the following holds.

1. $\psi \in C^{1}(E ; \mathbb{R}), \psi^{\prime}=g \in C\left(E ; E^{\star}\right)$ and $\psi$ and $\psi^{\prime}$ are bounded on bounded sets.

2. $\forall(u, v) \in E \times E,\langle g(u), v\rangle_{E^{\star}, E}=\operatorname{Re} \int_{\mathbb{R}^{N}} g(u)(x) \overline{v(x)} \mathrm{d} x$.

3. Let $\left(u_{n}\right)_{n},\left(v_{n}\right)_{n} \subset E$ be bounded. If $\lim _{n \rightarrow \infty}\left\|u_{n}-v_{n}\right\|_{L^{p}\left(\mathbb{R}^{N}\right)}=0$, for some $p \in[1, \infty]$, then $\lim _{n \rightarrow \infty}\left|\psi\left(u_{n}\right)-\psi\left(v_{n}\right)\right|=0$.

4. Let $u \in H^{1}\left(\mathbb{R}^{N}\right)$. If $u_{n} \frac{H_{\mathrm{w}}^{1}}{n \rightarrow \infty} u$ then $g\left(u_{n}\right) \underset{n \rightarrow \infty}{\stackrel{E_{\mathrm{w}}^{\star}}{\longrightarrow}} g(u)$.

For more details, see the proof of Proposition 5.1 in the Appendix A.

From now and until the end of this section, we shall suppose that Assumptions 1.1 and 1.3 are fulfilled. In particular, by (1.10), $g \not \equiv 0$. Note that when $g \equiv 0$ then by Remark $2.8, u \equiv 0$ is the unique solution to $(1.19)$.

Let for any $t \geqslant 0, \mathbb{S}_{t} \stackrel{\text { def }}{=}\left\{u \in \mathscr{D}\left(\mathbb{R}^{N}\right) ;\|u\|_{H_{A, V}^{1}\left(\mathbb{R}^{N}\right)}^{2}=t\right\}, \overline{\mathbb{S}_{t}} \stackrel{\text { def }}{=}\left\{u \in H_{A, V}^{1}\left(\mathbb{R}^{N}\right) ;\|u\|_{H_{A, V}^{1}\left(\mathbb{R}^{N}\right)}^{2}=t\right\}$, $\overline{B_{t}} \stackrel{\text { def }}{=}\left\{u \in H_{A, V}^{1}\left(\mathbb{R}^{N}\right) ;\|u\|_{H_{A, V}^{1}\left(\mathbb{R}^{N}\right)}^{2} \leqslant t\right\}$ and

$$
\gamma(t) \stackrel{\text { def }}{=} \sup _{u \in \overline{\mathbb{S}_{t}}} \psi(u)=\sup _{u \in \mathbb{S}_{t}} \psi(u)
$$

where the second equality in (5.1) comes from density of $\mathscr{D}\left(\mathbb{R}^{N}\right)$ in $H_{A, V}^{1}\left(\mathbb{R}^{N}\right)$ (Theorem 2.3). Furthermore let

$$
I_{\gamma} \stackrel{\text { def }}{=}\left(2 \inf _{t \neq s} \frac{\gamma(t)-\gamma(s)}{t-s}, 2 \sup _{t \neq s} \frac{\gamma(t)-\gamma(s)}{t-s}\right) \text {, }
$$

and for any $\rho>0$,

$$
\begin{gathered}
\forall u \in H_{A, V}^{1}\left(\mathbb{R}^{N}\right), G_{\rho}(u) \stackrel{\text { def }}{=} \frac{\rho}{2}\|u\|_{H_{A, V}^{1}}^{2}\left(\mathbb{R}^{N}\right)-\psi(u), \\
\forall t \geqslant 0, \Gamma_{\rho}(t) \stackrel{\text { def }}{=} \frac{\rho}{2} t-\gamma(t) .
\end{gathered}
$$

Note that by Proposition 5.1 and Theorems 2.3 and 2.7, $G_{\rho} \in C^{1}\left(H_{A, V}^{1}\left(\mathbb{R}^{N}\right) ; \mathbb{R}\right)$ and

$$
G_{\rho}^{\prime}(u)=\rho\left(-\Delta_{A} u+V u\right)-g(u), \text { in } H_{A, V}^{-1}\left(\mathbb{R}^{N}\right) .
$$

It follows that for $\lambda=\frac{1}{\rho}, u_{\rho}$ is a weak solution to (1.19) if, and only if, $G_{\rho}^{\prime}\left(u_{\rho}\right)=0$. 
Lemma 5.3. The function $\gamma$ defined by (5.1) is continuous and nondecreasing over $[0, \infty)$ and is locally Lipschitz continuous over $(0, \infty)$. Furthermore, $\gamma$ has a derivative at $t=0$ and $\gamma^{\prime}(0)=0$. In addition, for any $a \geqslant 0$ and $b \geqslant 0$,

$$
\gamma(a)+\gamma(b) \leqslant \gamma(a+b) .
$$

Finally, $I_{\gamma} \neq \emptyset$ and $I_{\gamma}=\left(0,2 \sup _{t \neq s} \frac{\gamma(t)-\gamma(s)}{t-s}\right)$.

Proof. Let $u \in H_{A, V}^{1}\left(\mathbb{R}^{N}\right)$.

Let $\theta>0$. Let $\left(v_{k}\right)_{k \in \mathbb{N}} \subset \mathbb{S}_{1}$ be such that $v_{k} \longrightarrow 0$ in $H_{A, V^{-}}^{1}$ weakly and $\operatorname{supp} v_{k} \subset Q$. By Theorem 2.3 and compactness, $v_{k} \underset{k \rightarrow \infty}{\stackrel{H_{w}^{1}}{\longrightarrow}} 0$ and $v_{k} \underset{k \rightarrow \infty}{\stackrel{L^{p}\left(\mathbb{R}^{N}\right)}{\longrightarrow}} 0$, for any $p \in\left(2,2^{\star}\right)$, It follows from Property 3 of Proposition 5.1 that,

$$
\psi\left(u+\theta v_{k}\right) \stackrel{k \rightarrow \infty}{\longrightarrow} \psi(u) \text { and }\left\|u+\theta v_{k}\right\|_{H_{A, V}^{1}\left(\mathbb{R}^{N}\right)}^{2} \stackrel{k \rightarrow \infty}{\longrightarrow}\|u\|_{H_{A, V}^{1}\left(\mathbb{R}^{N}\right)}^{2}+\theta^{2} .
$$

Let $t>0$. Let $\left(u_{k}\right)_{k \in \mathbb{N}} \subset \mathbb{S}_{t}$ be a such that $\psi\left(u_{k}\right) \stackrel{k \rightarrow \infty}{\longrightarrow} \gamma(t)$ and $\operatorname{supp} u_{k} \subset B\left(0, R_{k}\right)$. Since $\mathscr{D}\left(\mathbb{R}^{N}\right)$ is dense in $H_{A, V}^{1}\left(\mathbb{R}^{N}\right)$ (Theorem 2.3), we may find $\left(w_{k}\right)_{k \in \mathbb{N}} \subset \mathscr{D}\left(\mathbb{R}^{N}\right)$ such that supp $w_{k} \subset B\left(0, r_{k}\right)$ and $w_{k} \underset{k \rightarrow \infty}{\stackrel{H_{A, V}^{1}\left(\mathbb{R}^{N}\right)}{\longrightarrow}} u$. Let $\left(y_{k}\right)_{k} \subset \mathbb{Z}^{N}$ with $\left|y_{k}\right|>R_{k}+r_{k}$ and let $v_{k}=\tau_{y_{k}} u_{k} \in H_{A, V}^{1}\left(\mathbb{R}^{N}\right)$. It follows that,

$$
\forall k \in \mathbb{N}, \operatorname{supp} v_{k} \cap \operatorname{supp} w_{k}=\emptyset,
$$

from which we deduce for any $k \in \mathbb{N}, \psi\left(v_{k}+w_{k}\right)=\psi\left(v_{k}\right)+\psi\left(w_{k}\right)$. By Theorem 2.3, Proposition 5.1 and the fact that $\psi$ is invariant with respect to $D$, we have for any $k \in \mathbb{N}$,

$$
\begin{aligned}
& \left|\psi\left(u+v_{k}\right)-(\psi(u)+\gamma(t))\right| \\
\leqslant & \left|\psi\left(u+v_{k}\right)-\psi\left(w_{k}+v_{k}\right)\right|+\left|\psi\left(w_{k}\right)-\psi(u)\right|+\left|\psi\left(v_{k}\right)-\gamma(t)\right| \\
\leqslant & C\left\|w_{k}-u\right\|_{H_{A, V}^{1}\left(\mathbb{R}^{N}\right)}+\left|\psi\left(u_{k}\right)-\gamma(t)\right| \stackrel{k \rightarrow \infty}{\longrightarrow} 0 .
\end{aligned}
$$

It follows that,

$$
\lim _{k \rightarrow \infty} \psi\left(u+v_{k}\right)=\psi(u)+\gamma(t) .
$$

Finally, by (5.7) and Cauchy-Schwarz's inequality,

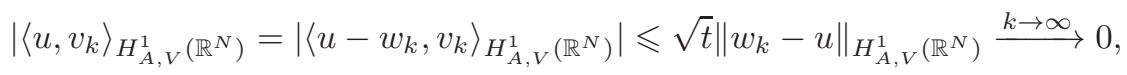

from which we get with help of (3.11),

$$
\lim _{k \rightarrow \infty}\left\|u+v_{k}\right\|_{H_{A, V}^{1}\left(\mathbb{R}^{N}\right)}^{2}=\|u\|_{H_{A, V}^{1}\left(\mathbb{R}^{N}\right)}^{2}+t .
$$


And since $\psi^{\prime}$ is bounded on bounded sets (Proposition 5.1), we conclude with (2.6) and (2.7) that there exists $C_{t}>0$ such that,

$$
\forall u \in \overline{B_{t}},\left|\left\langle\psi^{\prime}(u), u\right\rangle_{H_{A, V}^{-1}\left(\mathbb{R}^{N}\right), H_{A, V}^{1}\left(\mathbb{R}^{N}\right)}\right| \leqslant C_{t} .
$$

By (5.6), (5.8)-(5.10) and [10, Theorem 2.1], it follows that $\gamma$ is locally Lipschitz continuous and nondecreasing over $(0, \infty)$ and $(5.5)$ holds true. Now, let us prove that $\gamma^{\prime}(0)=\inf _{t \neq s} \frac{\gamma(t)-\gamma(s)}{t-s}=0$. Let $\varepsilon>0$. We let $\kappa=1$, if $N \geqslant 3$ and $\kappa=0$, if $N=2$. Let $C_{\varepsilon}>0$ and $p_{\varepsilon}>2$ be given by (1.8)-(1.9). By (2.6) and the Sobolev embeddings, there exist $C>0$, which does not depend on $\varepsilon$, and $C_{\varepsilon}^{\prime}>0$ such that for any $t>0$,

$$
\begin{aligned}
0 & \leqslant \inf _{t \neq s} \frac{\gamma(t)-\gamma(s)}{t-s} \leqslant \frac{\gamma(t)-\gamma(0)}{t} \leqslant \frac{1}{t} \sup _{u \in \mathbb{S}_{t}} \int_{\mathbb{R}^{N}}|F(x,|u|)| \mathrm{d} x \\
& \leqslant \frac{1}{t} \sup _{u \in \mathbb{S}_{t}}\left(\varepsilon \int_{\mathbb{R}^{N}}\left(|u|^{2}+\kappa|u|^{2^{\star}}\right) \mathrm{d} x+C_{\varepsilon} \int_{\mathbb{R}^{N}}|u|^{p_{\varepsilon}} \mathrm{d} x\right) \\
& \leqslant \frac{C \varepsilon}{t}\left(\sup _{u \in \mathbb{S}_{t}}\|u\|_{\left.H_{A, V}^{1} \mathbb{R}^{N}\right)}^{2}+\kappa \sup _{u \in \mathbb{S}_{t}}^{\|u\|_{H_{A, V}^{1}}^{2^{\star}}\left(\mathbb{R}^{N}\right)}\right)+\frac{C_{\varepsilon}^{\prime} C_{\varepsilon}}{t} \sup _{u \in \mathbb{S}_{t}}\|u\|_{H_{A, V}^{1}}^{p_{\varepsilon}}\left(\mathbb{R}^{N}\right) \\
& \leqslant C \varepsilon\left(1+\kappa t^{\frac{2^{\star}-2}{2}}\right)+C_{\varepsilon}^{\prime} C_{\varepsilon} t^{\frac{p_{\varepsilon}-2}{2}} \stackrel{t \searrow 0}{\longrightarrow} C \varepsilon .
\end{aligned}
$$

Since $\varepsilon$ is arbitrary, we can conclude that $\inf _{t \neq s} \frac{\gamma(t)-\gamma(s)}{t-s}=\gamma^{\prime}(0)=\lim _{t \searrow 0} \frac{\gamma(t)-\gamma(0)}{t}=0$. Finally, if $I_{\gamma}$ where empty then we would have for any $t \geqslant 0, \gamma(t)=0$. But this would yield $\psi(u) \leqslant 0$, for any $u \in H^{1}\left(\mathbb{R}^{N}\right)$, contradicting (1.10).

We shall use the well-known following result.

Theorem 5.4 ([14], Theorem 3.1, p.62-63). Let $\left(u_{k}\right)_{k \in \mathbb{N}} \subset H_{A, V}^{1}\left(\mathbb{R}^{N}\right)$ be a bounded sequence and let $t_{0} \stackrel{\text { def }}{=} \limsup _{k \rightarrow \infty}\left\|u_{k}\right\|_{H_{A, V}^{1}}^{2}\left(\mathbb{R}^{N}\right)$. Then, up to subsequence that we will still denote by $\left(u_{k}\right)_{k \in \mathbb{N}}$, there exist $\mathbb{D} \subset \mathbb{N},\left(w^{n}\right)_{n \in \mathbb{D}} \subset H_{A, V}^{1}\left(\mathbb{R}^{N}\right)$ and $\left(\tau_{y_{k}^{n}}\right)_{(k, n) \in \mathbb{N} \times \mathbb{D}} \subset D$ such that for any $(n, m) \in \mathbb{D} \times \mathbb{D}$,

$$
\begin{aligned}
& \tau_{-y_{k}^{n}} u_{k} \underset{k \rightarrow \infty}{\longrightarrow} w^{n}, \text { in } H_{A, V^{-}}^{1} \text { weakly, } \\
& \lim _{k \rightarrow \infty}\left|y_{k}^{m}-y_{k}^{n}\right|=\infty, \text { for } n \neq m, \\
& \sum_{n \in \mathbb{D}}\left\|w^{n}\right\|_{\left.H_{A, V}^{1} \mathbb{R}^{N}\right)}^{2} \leqslant t_{0} \\
& u_{k}-\sum_{n \in \mathbb{D}} \tau_{y_{k}^{n}} w^{n} \frac{D}{k \rightarrow \infty} 0
\end{aligned}
$$

where the series in (5.14) converges uniformly in $k \in \mathbb{N}$.

Proof. Since $H_{A, V}^{1}\left(\mathbb{R}^{N}\right)$ is a separable infinite-dimensional Hilbert space and $D$ is a set of dislocations on $H_{A, V}^{1}\left(\mathbb{R}^{N}\right)$ ((2.5) and Proposition 3.8) and using (3.10), we may apply [14, Theorem 3.1, p.62$63]$ which asserts, up to subsequence that we will still denote by $\left(u_{k}\right)_{k \in \mathbb{N}}$, the existence of $\mathbb{D} \subset \mathbb{N}$, 
$\left(w^{n}\right)_{n \in \mathbb{D}} \subset H_{A, V}^{1}\left(\mathbb{R}^{N}\right)$ and $\left(\tau_{y_{k}^{n}}\right)_{(k, n) \in \mathbb{N} \times \mathbb{D}} \subset \mathbb{D}$ satisfying (5.11), (5.13), (5.14) and $\tau_{-y_{k}^{n}} \tau_{y_{k}^{m}} \underset{k \rightarrow \infty}{\longrightarrow} 0$, for $n \neq m$. This last estimate and Lemma 3.7 yields (5.12).

Lemma 5.5. For almost every $\rho \in I_{\gamma}$, there exist $c(\rho)>0$ and a bounded critical sequence $\left(u_{k}\right)_{k \in \mathbb{N}} \subset$ $H_{A, V}^{1}\left(\mathbb{R}^{N}\right)$ that is,

$$
\begin{gathered}
\left(u_{k}\right)_{k \in \mathbb{N}} \subset H_{A, V}^{1}\left(\mathbb{R}^{N}\right) \text { is bounded, } \\
\left\{\begin{array}{l}
G_{\rho}\left(u_{k}\right) \stackrel{k \rightarrow \infty}{\longrightarrow} c(\rho)>0, \\
G_{\rho}^{\prime}\left(u_{k}\right) \stackrel{H_{A, V}^{-1}\left(\mathbb{R}^{N}\right)}{k \rightarrow \infty} 0 .
\end{array}\right.
\end{gathered}
$$

In addition, for every $\rho \in I_{\gamma}$, there exist $c(\rho)>0$ and a sequence $\left(u_{k}\right)_{k \in \mathbb{N}} \subset H_{A, V}^{1}\left(\mathbb{R}^{N}\right)$ satisfying (5.16).

Proof. The proof of [10, Theorem 2.15] can be adapted to prove Lemma 5.5. Let $\rho_{0} \in I_{\gamma}$. Then $\Gamma_{\rho_{0}}$ is not monotone nondecreasing. Indeed, if so then for any $t_{1}<t_{2}$ we would have

$$
\frac{\rho_{0}}{2} t_{1}-\gamma\left(t_{1}\right) \leqslant \frac{\rho_{0}}{2} t_{2}-\gamma\left(t_{2}\right)
$$

which implies $2 \sup _{t \neq s} \frac{\gamma(t)-\gamma(s)}{t-s} \leqslant \rho_{0}$, contradicting the fact that $\rho_{0} \in I_{\gamma}$. (Similarly $\Gamma_{\rho_{0}}$ is not monotone nonincreasing.) Therefore, we can find $0<t_{0}<t_{1}$ and a $\delta>0$ such that $\Gamma_{\rho_{0}}\left(t_{0}\right)>\Gamma_{\rho_{0}}\left(t_{1}\right)+3 \delta>3 \delta$ (we recall that by Lemma $5.3, \Gamma_{\rho_{0}}(0)=0$ and $\Gamma_{\rho_{0}}^{\prime}(0)>0$ ). Also, it is clear that the mapping $\rho \longmapsto \Gamma_{\rho}\left(t_{0}\right)-\Gamma_{\rho}\left(t_{1}\right)$ is continuous over $[0, \infty)$ so that there exists $\delta_{0}\left(\rho_{0}\right)>0$ such that for any $\rho \in I_{\rho_{0}} \stackrel{\text { def }}{=}\left(\rho_{0}-\delta_{0}\left(\rho_{0}\right), \rho_{0}+\delta_{0}\left(\rho_{0}\right)\right), \Gamma_{\rho}\left(t_{0}\right)>\Gamma_{\rho}\left(t_{1}\right)+2 \delta>2 \delta$. But it follows from the definition of $\gamma$ that there is a $u_{1} \in \mathbb{S}_{t_{1}}$ such that $\psi\left(u_{1}\right)>\gamma\left(t_{1}\right)-\delta$. Thus, for any $\rho \in I_{\rho_{0}}$ and any $u \in \overline{\mathbb{S}_{t_{0}}}$,

$$
G_{\rho}(u) \geqslant \Gamma_{\rho}\left(t_{0}\right)>\Gamma_{\rho}\left(t_{1}\right)+2 \delta>G_{\rho}\left(u_{1}\right)+\delta .
$$

Denoting by $\Lambda \stackrel{\text { def }}{=}\left\{\xi \in C\left([0,1] ; H_{A, V}^{1}\left(\mathbb{R}^{N}\right)\right) ; \xi(0)=0\right.$ and $\left.\xi(1)=u_{1}\right\}$, it follows from (5.17) that the following holds.

$$
\left\{\begin{array}{l}
\text { For any } \rho_{0} \in I_{\gamma}, \text { there exist } \delta_{0}\left(\rho_{0}\right)>0 \text { and } u_{1} \in H_{A, V}^{1}\left(\mathbb{R}^{N}\right) \backslash\{0\} \\
\text { such that for any } \rho \in I_{\rho_{0}} \stackrel{\text { def }}{=}\left(\rho_{0}-\delta_{0}\left(\rho_{0}\right), \rho_{0}+\delta_{0}\left(\rho_{0}\right)\right), \\
c(\rho) \stackrel{\text { def }}{=} \inf _{\xi \in \Lambda} \max _{t \in[0,1]} G_{\rho}(\xi(t))>G_{\rho}\left(u_{1}\right)>G_{\rho}(0) .
\end{array}\right.
$$

Thus $G_{\rho_{0}}$ has mountain pass geometry and we can find a critical sequence satisfying (5.16) by the Mountain Pass Theorem (see, for instance, [14, Theorem 6.2, p.144]). Now, let us show that for almost every $\rho \in I_{\gamma}$, there exists a bounded critical sequence. As we shall see, this is almost a direct 
consequence of (5.18) and [6, Theorem 1.1] (see also [9, 13]). Because of the form of the functional $G_{\rho}$, we cannot directly apply [6]. But it can be easily adapted and we postpone its proof to the Appendix A (see Theorem A.1 below). Let $I \subset(0, \infty)$ be any interval. Let us consider the following Property $\left(P_{I}\right)$.

$$
\left\{\begin{array}{l}
\text { For almost every } \rho \in I, \text { there exists a sequence } \\
\left(u_{k}\right)_{k \in \mathbb{N}} \subset H_{A, V}^{1}\left(\mathbb{R}^{N}\right) \text { satisfying (5.15)-(5.16). }
\end{array}\right.
$$

Let $\left(a_{n}\right)_{n \in \mathbb{N}} \subset I_{\gamma}$ be any increasing sequence converging towards $\sup I_{\gamma}$. For each $n \in \mathbb{N}$, let $I_{n} \stackrel{\text { def }}{=}\left(\frac{1}{n}, a_{n}\right)$. Let $n \in \mathbb{N}$ be such that $I_{n} \neq \emptyset$. By (5.18) and Theorem A.1, for each $\rho_{0} \in I_{\gamma}$, $I_{\rho_{0}}$ satisfies $\left(P_{I_{\rho_{0}}}\right)$. But $\overline{I_{n}} \subset \bigcup_{\rho_{0} \in I_{\gamma}} I_{\rho_{0}}$ and by compactness, $I_{n}$ may be covered by a finite number of $I_{\rho_{0}}$. Consequently, $I_{n}$ satisfies $\left(P_{I_{n}}\right)$. Since $n \in \mathbb{N}$, is arbitrary, we infer that $I_{\gamma}=\bigcup_{n \in \mathbb{N}} I_{n}$ satisfies $\left(P_{I_{\gamma}}\right)$. This ends the proof of the lemma.

Corollary 5.6. For almost every $\rho \in I_{\gamma}$, there exists $u_{\rho} \in H_{A, V}^{1}\left(\mathbb{R}^{N}\right) \backslash\{0\}$ such that $G_{\rho}^{\prime}\left(u_{\rho}\right)=0$. In particular, $u_{\rho}$ is a non zero weak solution to (1.19) with $\lambda=\frac{1}{\rho}$.

Proof. By Lemma 5.5, for almost every $\rho \in I_{\gamma}$, there exist $c(\rho)>0$ and a sequence $\left(u_{k}\right)_{k \in \mathbb{N}} \subset$ $H_{A, V}^{1}\left(\mathbb{R}^{N}\right)$ satisfying (5.15)-(5.16). Let such $\rho, c \stackrel{\text { def }}{=} c(\rho)$ and $\left(u_{k}\right)_{k \in \mathbb{N}}$. We first extract a subsequence

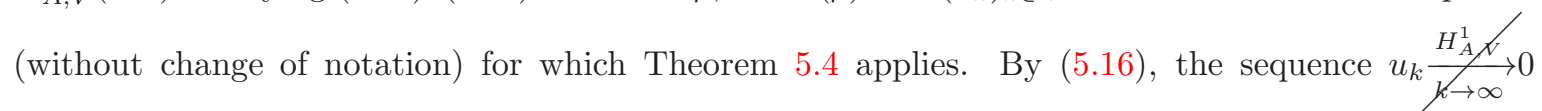
because $c>0$ and $G_{\rho}(0)=0$. Thus we may assume that, up to a subsequence that we still denote by $\left(u_{k}\right)_{k \in \mathbb{N}},\left\|u_{k}\right\|_{H_{A, V}^{1}\left(\mathbb{R}^{N}\right)}^{2} \longrightarrow t>0$. It follows from (5.16) that $\left\langle G_{\rho}^{\prime}\left(u_{k}\right), u_{k}\right\rangle_{H_{A, V}^{-1}, H_{A, V}^{1}} \longrightarrow 0$. If $u_{k} \stackrel{D}{\longrightarrow} 0$ then (2.9), Proposition 5.1, (1.8)-(1.9), Hölder's inequality, Sobolev's embedding and Theorem 4.1 imply that for any $\varepsilon>0$, there is a $p_{\varepsilon} \in\left(2,2^{\star}\right)$ such that for some $C_{\varepsilon}>0$,

$$
\begin{aligned}
& \left|\left\langle g\left(u_{k}\right), u_{k}\right\rangle_{H_{A, V}^{-1}, H_{A, V}^{1}}\right| \leqslant \int_{\mathbb{R}^{N}}\left|g\left(u_{k}\right) \| u_{k}\right| \mathrm{d} x \\
\leqslant & C\left(\sup _{k \in \mathbb{N}}\left\|u_{k}\right\|_{H_{A, V}^{1}\left(\mathbb{R}^{N}\right)}\right) \varepsilon+C_{\varepsilon}\left\|u_{k}\right\|_{L^{p_{\varepsilon}\left(\mathbb{R}^{N}\right)}}^{p_{\varepsilon}} \stackrel{k \rightarrow \infty}{\longrightarrow} C \varepsilon .
\end{aligned}
$$

But then, $\left\langle G_{\rho}^{\prime}\left(u_{k}\right), u_{k}\right\rangle_{H_{A, V}^{-1}, H_{A, V}^{1}} \longrightarrow \rho t \neq 0$, a contradiction. Then,

$$
u_{k} \stackrel{D}{\longrightarrow} 0, \text { as } k \longrightarrow \infty
$$

Let us apply and use the notations of Theorem 5.4. If $\mathbb{D}=\emptyset$ or if all the $w^{n}$ were zero, then by (5.14) we would have $u_{k} \stackrel{D}{\longrightarrow} 0$, contradicting (5.19). Therefore, $\mathbb{D} \neq \emptyset$ and there is at least one nonzero $w^{n_{0}}$ which we call $u_{\rho}$. Since for any $(k, n) \in \mathbb{N} \times \mathbb{D}$,

$$
G_{\rho}\left(\tau_{-y_{k}^{n}} u_{k}\right)=G_{\rho}\left(u_{k}\right) \text { and }\left\|G_{\rho}^{\prime}\left(\tau_{-y_{k}^{n}} u_{k}\right)\right\|_{H_{A, V}^{-1}\left(\mathbb{R}^{N}\right)}=\left\|G_{\rho}^{\prime}\left(u_{k}\right)\right\|_{H_{A, V}^{-1}\left(\mathbb{R}^{N}\right)}
$$


we conclude from (5.16), (5.11), Theorem 2.3 and Proposition 5.1 that,

$$
G_{\rho}^{\prime}\left(\tau_{-y_{k}^{n_{0}}} u_{k}\right) \underset{k \rightarrow \infty}{\longrightarrow} G_{\rho}^{\prime}\left(u_{\rho}\right)=0, \text { in } H_{A, V^{-}}^{-1} \text { weakly }
$$

from which the result follows.

Proof of Theorem 1.10. Apply Corollary 5.6 with $\rho=\frac{1}{\lambda}$.

\section{Applications}

In this section, we give some examples of nonlinearities for which Corollary 5.6 applies: for almost every $\lambda>0$ such that $\frac{1}{\lambda} \in I_{\gamma}=(0,2 S)$, where $S \stackrel{\text { def }}{=} \sup _{t \neq s} \frac{\gamma(t)-\gamma(s)}{t-s} \in(0, \infty]$, there exists, at least, a non zero weak solution to (1.19).

Example 6.1 (The single power interaction). Let $1<p<2^{\star}-1$ and let,

$$
\forall u \in H^{1}\left(\mathbb{R}^{N}\right), g(u)=|u|^{p-1} u .
$$

Then (1.8)-(1.10) are satisfied and Corollary 5.6 applies. It is not hard to see that $\frac{\gamma(t)}{t} \stackrel{t \rightarrow \infty}{\longrightarrow} \infty$ so that $I_{\gamma}=(0, \infty)$. Let $\lambda>0$. Let then $\lambda_{0} \in I_{\gamma}$ for which (1.19) admits a non zero weak solution $u_{\lambda_{0}}$. Setting $u=\left(\frac{\lambda}{\lambda_{0}}\right)^{\frac{1}{p-1}} u_{\lambda_{0}}$, a straightforward calculation shows that $u$ is a solution to (1.19) with $\lambda g(u)$ as the right side. In conclusion, for any $\lambda>0$, equation (1.19) has, at least, a non zero weak solution. Note that $F$ satisfies the Rabinowitz condition.

Example 6.2 (The combined power-type interaction). Let $\mu_{1}, \mu_{2}>0$, let $1<p_{1} \neq p_{2}<2^{\star}-1$ and let,

$$
\forall u \in H^{1}\left(\mathbb{R}^{N}\right), g(u)=\mu_{1}|u|^{p_{1}-1} u-\mu_{2}|u|^{p_{2}-1} u .
$$

The only difficulty is to show that there is a $u \in H^{1}\left(\mathbb{R}^{N}\right)$ such that,

$$
\psi(u) \stackrel{\text { def }}{=} \frac{\mu_{1}}{p_{1}+1}\|u\|_{L^{p_{1}+1}\left(\mathbb{R}^{N}\right)}^{p_{1}+1}-\frac{\mu_{2}}{p_{2}+1}\|u\|_{L^{p_{2}+1}\left(\mathbb{R}^{N}\right)}^{p_{2}+1}>0 .
$$

Let $u \in H_{A, V}^{1}\left(\mathbb{R}^{N}\right) \backslash\{0\}$ and let $t>0$. If $p_{1}<p_{2}$ then

$$
\psi(t u)=t^{p_{1}+1}\left(\frac{\mu_{1}}{p_{1}+1}\|u\|_{L^{p_{1}+1}\left(\mathbb{R}^{N}\right)}^{p_{1}+1}-\frac{\mu_{2}}{p_{2}+1} t^{p_{2}-p_{1}}\|u\|_{L^{p_{2}+1}\left(\mathbb{R}^{N}\right)}^{p_{2}+1}\right)>0,
$$

for any $0<t \ll 1$, while if $p_{1}>p_{2}$ then

$$
\psi(t u)=t^{p_{2}+1}\left(\frac{\mu_{1}}{p_{1}+1} t^{p_{1}-p_{2}}\|u\|_{L^{p_{1}+1}\left(\mathbb{R}^{N}\right)}^{p_{1}+1}-\frac{\mu_{2}}{p_{2}+1}\|u\|_{L^{p_{2}+1}\left(\mathbb{R}^{N}\right)}^{p_{2}+1} \stackrel{t \rightarrow \infty}{\longrightarrow} \infty .\right.
$$


Then (1.8)-(1.10) are satisfied and Corollary 5.6 applies. In particular, it follows from the last estimate that if $p_{1}>p_{2}$ then $\frac{\gamma(t)}{t} \stackrel{t \rightarrow \infty}{\longrightarrow} \infty$ so that $I_{\gamma}=(0, \infty)$ and we may choose $\lambda$ as close to 1 as we want. Notice also that $\psi<0$ on a nonempty open subset which is very different from the most hypotheses that can be found in the literature (as the Rabinowitz condition, for instance).

Example 6.3. Suppose that $F$ does not satisfy the Rabinowitz condition: $F(x, t) \geqslant \mu t f(x, t)>0$ with $\mu>2$ but there are an $M>0$ and a $c>0$ such that for any $t>M, F(x, t) \geqslant c t^{2} \ln t$. Then

$$
\begin{aligned}
\lim _{t \rightarrow \infty} \frac{\gamma(t)}{t} & \geqslant c \lim _{t \rightarrow \infty} \sup _{u \in \mathbb{S}_{t}} \frac{1}{t} \int_{\mathbb{R}^{N}}|u|^{2} \ln |u| \mathrm{d} x \\
& =c \lim _{t \rightarrow \infty} \sup _{u \in \mathbb{S}_{1}} \frac{1}{t} \int_{\mathbb{R}^{N}} t|u|^{2} \ln (\sqrt{t}|u|) \mathrm{d} x \\
& =\infty
\end{aligned}
$$

So that $I_{\gamma}=(0, \infty)$ and Corollary 5.6 applies for almost every $\lambda>0$. As an example of $g$ satisfying such a condition and (1.8)-(1.10) is,

$$
\forall u \in H^{1}\left(\mathbb{R}^{N}\right), g(u)= \begin{cases}c_{\varepsilon}|u|^{p-1} u, & \text { if }|u|<\varepsilon, \\ \mu_{1} u \ln |u|+\mu_{2} u, & \text { if }|u| \geqslant \varepsilon,\end{cases}
$$

where $\mu_{1}, \mu_{2}, \varepsilon>0$ and $p \in\left(1,2^{\star}-1\right)$ can be chosen arbitrarily and $c_{\varepsilon}=\varepsilon^{-(p-1)}\left(\mu_{1} \ln \varepsilon+\mu_{2}\right)$.

\section{Appendix}

\section{A Some proofs}

In this appendix, we adapt the proof of [6, Theorem 1.1] to our family of functionals $\left(G_{\rho}\right)_{\rho \in I_{\gamma}}$, where the original idea is due to [12]. We also give the proof of Proposition 5.1.

In [6], the family of functionals is of the form

$$
\forall \lambda>0, I_{\lambda}(u)=A(u)-\lambda B(u)
$$

where $A(u) \stackrel{\|u\| \rightarrow \infty}{\longrightarrow} \infty$ or $B(u) \stackrel{\|u\| \rightarrow \infty}{\longrightarrow} \infty$, and with $B \geqslant 0$ everywhere. Unfortunately, in our case,

$$
\forall \rho>0, G_{\rho}(u)=\frac{1}{\lambda} I_{\lambda}(u)=\frac{1}{\lambda}\left(\frac{\|u\|^{2}}{2}-\lambda \psi(u)\right), \quad \lambda=\frac{1}{\rho},
$$

and we do not have $B=\psi \geqslant 0$, everywhere, but only somewhere. So we have, in some sense, to reverse the role of $A=\|u\|^{2}$ and $B=\psi$. The following theorem is an easy adaptation of [6, Theorem 1.1], but for the convenience of the reader, we give its proof. 
Theorem A.1 ([6], Theorem 1.1). Let $(X,\|\cdot\|)$ be a Banach space, let $I \subset(0, \infty)$ be a nonempty open interval and let $\left(G_{\rho}\right)_{\rho \in I} \subset C^{1}(X ; \mathbb{R})$ be a family of functionals of the form,

$$
\forall \rho \in I, G_{\rho}(u)=\rho A(u)-B(u)
$$

where $A \not \equiv 0$ and for any $u \in X, A(u) \geqslant 0$. Assume that either $A(u) \stackrel{\|u\| \rightarrow \infty}{\longrightarrow} \infty$ or $B(u) \stackrel{\|u\| \rightarrow \infty}{\longrightarrow} \infty$. We also assume that $\left(G_{\rho}\right)_{\rho \in I}$ has mountain pass geometry: there exist $u_{1} \in X$ and $u_{2} \in X$ such that, denoting by

$$
\Gamma \stackrel{\text { def }}{=}\left\{\xi \in C([0,1] ; X) ; \xi(0)=u_{1} \text { and } \xi(1)=u_{2}\right\}
$$

the set of continuous paths joining $u_{1}$ to $u_{2}$, we have for any $\rho \in I$,

$$
c(\rho) \stackrel{\text { def }}{=} \inf _{\xi \in \Gamma} \max _{t \in[0,1]} G_{\rho}(\xi(t))>\max \left\{G_{\rho}\left(u_{1}\right), G_{\rho}\left(u_{2}\right)\right\} .
$$

Then for almost every $\rho \in I, G_{\rho}$ admits a bounded Palais-Smale sequence: there exists a sequence $\left(u_{n}\right)_{n \in \mathbb{N}} \subset X$ satisfying,

$$
\begin{aligned}
& \left(u_{n}\right)_{n \in \mathbb{N}} \subset X \text { is bounded, } \\
& \left\{\begin{array}{l}
G_{\rho}\left(u_{n}\right) \underset{n \rightarrow \infty}{\longrightarrow} c(\rho), \\
G_{\rho}^{\prime}\left(u_{n}\right) \underset{n \rightarrow \infty}{\stackrel{X^{\star}}{\longrightarrow}} 0
\end{array}\right.
\end{aligned}
$$

where $X^{\star}$ denotes the topological space of $X$.

Remark A.2. Here are some comments of Theorem A.1.

1) If there exist $\rho \in I$ and $\left(u_{1}, u_{2}\right) \in X \times X$ satisfying (A.2) then it is well-known, by the Mountain Pass Theorem, that there exists a Palais-Smale sequence $\left(u_{n}\right)_{n \in \mathbb{N}} \subset X$ satisfying (A.4) (see, for instance, [14, Theorem 6.2, p.144]). The difficulty is to find such a bounded sequence.

2) The proof of Theorem A.1 relies on the existence of the derivative $c^{\prime}(\rho)$ of $c(\rho)$. Since $A \geqslant 0$, we have by (A.2) that the mapping $c: \rho \longmapsto c(\rho)$ is nondecreasing over $I$. It follows that $c$ has a derivative $c^{\prime}$ almost everywhere on $I$. In the original proof, the existence almost everywhere on $I$ of $c^{\prime}$ is ensured by the fact that the mapping $c: \rho \longmapsto c(\rho)$ is nonincreasing over $I$.

Before proceeding to the proof of Theorem A.1, let us pick any $\rho \in I$ such that the derivative $c^{\prime}(\rho)$ exists (see the item 2) in the above remark). Let then $\rho_{0} \in(0, \rho)$ be small enough to have $\left(\rho-\rho_{0}, \rho+\rho_{0}\right) \subset I$ and

$$
\forall \widetilde{\rho} \in\left(\rho-\rho_{0}, \rho+\rho_{0}\right),\left|\frac{c(\widetilde{\rho})-c(\rho)}{\widetilde{\rho}-\rho}-c^{\prime}(\rho)\right| \leqslant 1 .
$$


Now, let us choose $\left(\rho_{n}\right)_{n \in \mathbb{N}} \subset\left(\rho, \rho+\rho_{0}\right)$ be a decreasing sequence such that $\rho_{n} \stackrel{n \rightarrow \infty}{\longrightarrow} \rho$. Finally, since $A(u) \stackrel{\|u\| \rightarrow \infty}{\longrightarrow} \infty$ or $B(u) \stackrel{\|u\| \rightarrow \infty}{\longrightarrow} \infty$ there exists $M>10$ such that for any $u \in X$,

$$
\|u\|>M \Longrightarrow \max \{A(u), B(u)\}>\max \left\{c^{\prime}(\rho)+3,2 \rho\left(c^{\prime}(\rho)+4\right)-c(\rho)\right\} .
$$

We shall need of the two following lemmas.

Lemma A.3. There exists $\left(\xi_{n}\right)_{n \in \mathbb{N}} \subset \Gamma$ satisfying the following properties.

1) Let $t \in[0,1]$. If $n \in \mathbb{N}$ is such that $G_{\rho}\left(\xi_{n}(t)\right) \geqslant c(\rho)-\left(\rho_{n}-\rho\right)$ then $\left\|\xi_{n}(t)\right\| \leqslant M$.

2) $\forall n \in \mathbb{N}, \max _{t \in[0,1]} G_{\rho}\left(\xi_{n}(t)\right) \leqslant c(\rho)+\left(c^{\prime}(\rho)+2\right)\left(\rho_{n}-\rho\right)$.

Proof. Let $\left(\xi_{n}\right)_{n \in \mathbb{N}} \subset \Gamma$ be such that for any $n \in \mathbb{N}$,

$$
\max _{t \in[0,1]} G_{\rho_{n}}\left(\xi_{n}(t)\right) \leqslant c\left(\rho_{n}\right)+\left(\rho_{n}-\rho\right) .
$$

Let $t \in[0,1]$. Let $n \in \mathbb{N}$. We have by the hypothesis in 1 ), (A.7) and (A.5),

$$
A\left(\xi_{n}(t)\right)=\frac{G_{\rho_{n}}\left(\xi_{n}(t)\right)-G_{\rho}\left(\xi_{n}(t)\right)}{\rho_{n}-\rho} \leqslant \frac{c\left(\rho_{n}\right)-c(\rho)}{\rho_{n}-\rho}+2 \leqslant c^{\prime}(\rho)+3 .
$$

In addition, since for any $u \in X$, the mapping $\rho \longmapsto G_{\rho}(u)$ is nondecreasing, it follows from (A.8) and the hypothesis in 1),

$$
B\left(\xi_{n}(t)\right)=\rho_{n} A\left(\xi_{n}(t)\right)-G_{\rho_{n}}\left(\xi_{n}(t)\right) \leqslant 2 \rho\left(c^{\prime}(\rho)+4\right)-c(\rho)
$$

Hence $\left\|\xi_{n}(t)\right\| \leqslant M$, by (A.6), (A.8) and (A.9). To prove the second part of the lemma, we see that (A.5) implies,

$$
c\left(\rho_{n}\right) \leqslant c(\rho)+\left(c^{\prime}(\rho)+1\right)\left(\rho_{n}-\rho\right) .
$$

Finally, (A.7) and (A.10) yield,

$$
\max _{t \in[0,1]} G_{\rho}\left(\xi_{n}(t)\right) \leqslant \max _{t \in[0,1]} G_{\rho_{n}}\left(\xi_{n}(t)\right) \leqslant c(\rho)+\left(c^{\prime}(\rho)+2\right)\left(\rho_{n}-\rho\right) .
$$

This ends the proof of the lemma.

Lemma A.4. Define for any $\varepsilon>0$,

$$
F_{\varepsilon} \stackrel{\text { def }}{=}\left\{u \in X ;\|u\| \leqslant 2 M \text { and }\left|G_{\rho}(u)-c(\rho)\right| \leqslant \varepsilon\right\} .
$$

Then for any $\varepsilon>0, F_{\varepsilon} \neq \emptyset$ and $\inf _{u \in F_{\varepsilon}}\left\|G_{\rho}^{\prime}(u)\right\|_{X^{\star}}=0$. 
Proof. Let $\left(\xi_{n}\right)_{n \in \mathbb{N}} \subset \Gamma$ be given by Lemma A.3. Then for each $n \in \mathbb{N}$, there exists $t_{n} \in[0,1]$ such that $0 \leqslant G_{\rho}\left(\xi_{n}\left(t_{n}\right)\right)-c(\rho) \leqslant\left(c^{\prime}(\rho)+2\right)\left(\rho_{n}-\rho\right) \stackrel{n \rightarrow \infty}{\longrightarrow} 0$ and $\left\|\xi\left(t_{n}\right)\right\| \leqslant M$. We infer that for any $\varepsilon>0$, there exists $n_{0} \in \mathbb{N}$ large enough such that $\xi\left(t_{n_{0}}\right) \in F_{\varepsilon}$. Now, we note that it is sufficient to show the result for any $\varepsilon>0$ small enough. If the result does not hold then there exists $0<\varepsilon_{0}<\frac{c(\rho)-\max \left\{G_{\rho}\left(u_{1}\right), G_{\rho}\left(u_{2}\right)\right\}}{2}$ such that $\inf _{u \in F_{2 \varepsilon_{0}}}\left\|G_{\rho}^{\prime}(u)\right\|_{X^{*}} \geqslant 2 \varepsilon_{0}$. We then may apply a deformation lemma to affirm that there exists a homeomorphism $\eta: X \longrightarrow X$ satisfying the following properties.

$$
\begin{aligned}
& \text { If }\left|G_{\rho}(u)-c(\rho)\right|>2 \varepsilon_{0} \text { then } \eta(u)=u . \\
& \forall u \in X, G_{\rho}(\eta(u)) \leqslant G_{\rho}(u) . \\
& \text { If }\|u\| \leqslant M \text { and } G_{\rho}(u)<c(\rho)+\varepsilon_{0} \text { then } G_{\rho}(\eta(u))<c(\rho)-\varepsilon_{0} .
\end{aligned}
$$

See for instance [5, Theorem 4.2, p.38]. The assertion (A.12) is not directly stated in this theorem but in its proof p.39. Let $m \in \mathbb{N}$ be large enough to have,

$$
\rho_{m}-\rho<\left(c^{\prime}(\rho)+2\right)\left(\rho_{m}-\rho\right)<\varepsilon_{0} .
$$

By (A.11), $\eta\left(\xi_{m}\right) \in \Gamma$. Let $t \in[0,1]$.

- If $G_{\rho}\left(\xi_{m}(t)\right) \leqslant c(\rho)-\left(\rho_{m}-\rho\right)$ then by (A.12),

$$
G_{\rho}\left(\eta\left(\xi_{m}(t)\right)\right) \leqslant c(\rho)-\left(\rho_{m}-\rho\right) .
$$

- If $G_{\rho}\left(\xi_{m}(t)\right)>c(\rho)-\left(\rho_{m}-\rho\right)$ then by Lemma A.3 and (A.14), $\left\|\xi_{m}(t)\right\| \leqslant M$ and $G_{\rho}\left(\xi_{m}(t)\right)<$ $c(\rho)+\varepsilon_{0}$. It then follows from (A.13) and (A.14),

$$
G_{\rho}\left(\eta\left(\xi_{m}(t)\right)\right)<c(\rho)-\varepsilon_{0}<c(\rho)-\left(\rho_{m}-\rho\right) .
$$

It follows from (A.15) and (A.16) that,

$$
c(\rho)=\inf _{\xi \in \Gamma} \max _{t \in[0,1]} G_{\rho}(\xi(t)) \leqslant \max _{t \in[0,1]} G_{\rho}\left(\eta\left(\xi_{m}(t)\right)\right) \leqslant c(\rho)-\left(\rho_{m}-\rho\right) .
$$

A contradiction, since $\rho_{m}-\rho>0$.

Proof of Theorem A.1. The result follows by applying Lemma A.4 with any sequence $\varepsilon_{n} \searrow 0$.

Proof of Proposition 5.1. Throughout this proof, we let $\kappa=1$, if $N \geqslant 3$ and $\kappa=0$, if $N \leqslant 2$. We will denote by $C_{1}>1$ and $p_{1}$ the constants given by (1.8)-(1.9) for $\varepsilon=1$. We proceed to the proof in 6 steps.

Step 1: $g: H^{1}\left(\mathbb{R}^{N}\right) \longrightarrow H^{-1}\left(\mathbb{R}^{N}\right)$ is well-defined, bounded on bounded sets and 2 holds. 
By (1.8)-(1.9), $g(u) \in L_{\text {loc }}^{1}\left(\mathbb{R}^{N}\right)$. Let $\varphi \in \mathscr{D}\left(\mathbb{R}^{N}\right)$. We have by (1.8)-(1.9), Hölder's inequality and the Sobolev embeddings,

$$
\begin{aligned}
& \quad\left|\langle g(u), \varphi\rangle_{\mathscr{D}^{\prime}\left(\mathbb{R}^{N}\right) ; \mathscr{D}\left(\mathbb{R}^{N}\right)}\right|=\left|\operatorname{Re} \int_{\mathbb{R}^{N}} g(u) \bar{\varphi} \mathrm{d} x\right| \\
& \leqslant C_{1}\left(\|u\|_{L^{2}\left(\mathbb{R}^{N}\right)}+\kappa\|u\|_{L^{2^{\star}\left(\mathbb{R}^{N}\right)}}^{2^{\star}-1}+\|u\|_{L^{p_{1}\left(\mathbb{R}^{N}\right)}}^{p_{1}}\right)\|\varphi\|_{H^{1}\left(\mathbb{R}^{N}\right)} \\
& \leqslant C\left(\|u\|_{H^{1}\left(\mathbb{R}^{N}\right)}+\kappa\|u\|_{H^{1}\left(\mathbb{R}^{N}\right)}^{2^{\star}-1}+\|u\|_{H^{1}\left(\mathbb{R}^{N}\right)}^{p_{1}-1}\right)\|\varphi\|_{H^{1}\left(\mathbb{R}^{N}\right)} .
\end{aligned}
$$

By density, it follows that $g: H^{1}\left(\mathbb{R}^{N}\right) \longrightarrow H^{-1}\left(\mathbb{R}^{N}\right)$ is well-defined, $g$ is bounded on bounded sets and Property 2 holds.

Step 2: $\psi \in C\left(H^{1}\left(\mathbb{R}^{N}\right) ; \mathbb{R}\right), \psi$ is bounded on bounded sets, Gâteaux-differentiable and its Gâteauxdifferential is $\psi_{\mathrm{g}}^{\prime}=g$.

Let $u \in H^{1}\left(\mathbb{R}^{N}\right)$. By (1.8)-(1.9), Hölder's inequality and the Sobolev embedding, $F(u) \in L^{1}\left(\mathbb{R}^{N} ; \mathbb{R}\right)$ so that $\psi: H^{1}\left(\mathbb{R}^{N}\right) \longrightarrow \mathbb{R}$ is well-defined and $\psi$ is bounded on bounded sets. Let $v \in H^{1}\left(\mathbb{R}^{N}\right)$. Still by (1.8)-(1.9), Hölder's inequality and the Sobolev embedding,

$$
\begin{gathered}
|\psi(u+v)-\psi(u)| \leqslant \int_{\mathbb{R}^{N}} \int_{|u|}^{|u+v|}\left(t+\kappa t^{2^{\star}-1}+C_{1} t^{p_{1}-1}\right) \mathrm{d} t \mathrm{~d} x \\
\leqslant C\left(\|u\|_{L^{2}}+\|v\|_{L^{2}}+\kappa\left(\|u\|_{L^{2^{\star}}}+\|v\|_{L^{2^{\star}}}\right)^{2^{\star}-1}+\left(\|u\|_{L^{p_{1}}}+\|v\|_{L^{p_{1}}}\right)^{p_{1}-1}\right)\|v\|_{H^{1}\left(\mathbb{R}^{N}\right)} .
\end{gathered}
$$

It follows that $\psi \in C\left(H^{1}\left(\mathbb{R}^{N}\right) ; \mathbb{R}\right)$. Let $v \in H^{1}\left(\mathbb{R}^{N}\right)$ and $0<|t|<1$. Since $u, v \in L^{2}\left(\mathbb{R}^{N}\right)$, the set

$$
\mathcal{N} \stackrel{\text { def }}{=}\left\{x \in \mathbb{R}^{N} ;|u(x)|=\infty \text { or }|v(x)|=\infty\right\}
$$

has Lebesgue measure 0 . Let $x \in \mathcal{N}^{c}$. If $u(x) \neq 0$ then using that

$$
|u(x)+t v(x)|=\sqrt{(u(x)+t v(x)) \overline{(u(x)+t v(x))}}>0,
$$

for $t$ small enough, we see that

$$
\frac{\mathrm{d}}{\mathrm{d} t} F(x,|u(x)+t v(x)|)_{\mid t=0}=\operatorname{Re}(\boldsymbol{f}(x, u(x)) \overline{v(x)}) .
$$

If $u(x)=0$ then by $(1.8)-(1.9)$,

$$
\left|\frac{F(x,|t v(x)|)-F(x, 0)}{t}\right| \leqslant C\left(|t||v(x)|^{2}+\kappa|t|^{2^{\star}-1}|v(x)|^{2^{\star}}+C_{1}|t|^{p_{1}-1}|v(x)|^{p_{1}}\right) \stackrel{t \rightarrow 0}{\longrightarrow} 0 .
$$

We then infer,

$$
\frac{F(.,|u+t v|)-F(.,|u|)}{t} \underset{t \longrightarrow 0}{\stackrel{\text { a.e. in } \mathbb{R}^{N}}{\longrightarrow}} \operatorname{Re}(\boldsymbol{f}(., u) \bar{v}) .
$$


By (1.8)-(1.9),

$$
\begin{aligned}
& \frac{F(.,|u+t v|)-F(.,|u|)-t \operatorname{Re}(f(x, u) \bar{v})}{t} \\
\leqslant & \frac{1}{t} \int_{|u|}^{|u+t v|}|f(., s)| \mathrm{d} s+|f(.,|u|)||v| \\
\leqslant & C\left(|u|+|v|+\kappa(|u|+|v|)^{2^{\star}-1}+(|u|+|v|)^{p_{1}-1}\right)|v| \in L^{1}\left(\mathbb{R}^{N}\right) .
\end{aligned}
$$

It follows from the dominated convergence Theorem and Property 2 that,

$$
\lim _{t \rightarrow 0} \frac{\psi(u+t v)-\psi(u)}{t}=\langle g(u), v\rangle_{H^{-1}\left(\mathbb{R}^{N}\right), H^{1}\left(\mathbb{R}^{N}\right)} .
$$

Hence Step 2.

Step 3: Let $u, v \in H^{1}\left(\mathbb{R}^{N}\right)$ and $\left(u_{n}\right)_{n \in \mathbb{N}} \subset H^{1}\left(\mathbb{R}^{N}\right)$ be bounded. Let $\varepsilon>0$. Choose $\varepsilon^{\prime}>0$ small enough to have,

$$
2 \varepsilon^{\prime}\left(\sup _{n \in \mathbb{N}}\left\|u_{n}\right\|_{L^{2}\left(\mathbb{R}^{N}\right)}^{2}+\|u\|_{L^{2}\left(\mathbb{R}^{N}\right)}^{2}+\kappa\left(\sup _{n \in \mathbb{N}}\left\|u_{n}\right\|_{L^{2^{\star}\left(\mathbb{R}^{N}\right)}}^{2^{\star}-1}+\|u\|_{L^{2^{\star}\left(\mathbb{R}^{N}\right)}}^{2^{\star}-1}\right)\right) \leqslant \varepsilon .
$$

For such an $\varepsilon^{\prime}$, let $p_{\varepsilon^{\prime}}$ and $C_{\varepsilon^{\prime}}$ be given by (1.8)-(1.9). For each $n \in \mathbb{N}$, let

$$
A_{n}=\left\{x \in \mathbb{R}^{N} ; \varepsilon^{\prime}\left(\left|u_{n}\right|+|u|+\kappa\left(\left|u_{n}\right|^{2^{\star}-1}+|u|^{2^{\star}-1}\right)\right) \leqslant C_{\varepsilon^{\prime}}\left(\left|u_{n}\right|^{p_{\varepsilon^{\prime}}-1}+|u|^{p_{\varepsilon^{\prime}}-1}\right)\right\} .
$$

It holds that,

$$
\left|\left\langle g\left(u_{n}\right)-g(u), v\right\rangle_{H^{-1}\left(\mathbb{R}^{N}\right), H^{1}\left(\mathbb{R}^{N}\right)}\right| \leqslant \int_{\mathbb{R}^{N}}\left|g\left(u_{n}\right)-g(u)\right||v| \mathbb{1}_{A_{n}} \mathrm{~d} x+\varepsilon\|v\|_{H^{1}\left(\mathbb{R}^{N}\right)} .
$$

Indeed, by (1.8)-(1.9), Hölder's inequality, the Sobolev embeddings and (A.17), we have,

$$
\begin{aligned}
& \left|\left\langle g\left(u_{n}\right)-g(u), v\right\rangle_{H^{-1}\left(\mathbb{R}^{N}\right), H^{1}\left(\mathbb{R}^{N}\right)}\right| \leqslant \int_{\mathbb{R}^{N}}\left|g\left(u_{n}\right)-g(u)\right||v| \mathrm{d} x \\
= & \int_{\mathbb{R}^{N}}\left|g\left(u_{n}\right)-g(u)\right||v| \mathbb{1}_{A_{n}} \mathrm{~d} x+\int_{\mathbb{R}^{N}}\left|g\left(u_{n}\right)-g(u)\right||v| \mathbb{1}_{A_{n}^{c}} \mathrm{~d} x \\
\leqslant & \int_{\mathbb{R}^{N}}\left|g\left(u_{n}\right)-g(u)\right||v| \mathbb{1}_{A_{n}} \mathrm{~d} x+2 \varepsilon^{\prime} \int_{\mathbb{R}^{N}}\left(\left|u_{n}\right|+|u|+\kappa\left(\left|u_{n}\right|^{2^{\star}-1}+|u|^{2^{\star}-1}\right)\right)|v| \mathrm{d} x \\
\leqslant & \int_{\mathbb{R}^{N}}\left|g\left(u_{n}\right)-g(u)\right||v| \mathbb{1}_{A_{n}} \mathrm{~d} x+\varepsilon\|v\|_{H^{1}\left(\mathbb{R}^{N}\right)} .
\end{aligned}
$$

Step 3 is proved.

Step 4: $\psi \in C^{1}\left(H^{1}\left(\mathbb{R}^{N}\right) ; \mathbb{R}\right)$ and $\psi^{\prime}=g$.

By Step 2, it remains to show that $g \in C\left(H^{1}\left(\mathbb{R}^{N}\right) ; H^{-1}\left(\mathbb{R}^{N}\right)\right)$ to have that $\psi$ is Fréchet-differentiable 
and $\psi^{\prime}=\psi_{\mathrm{g}}^{\prime}$. Assume $u_{n} \underset{k \rightarrow \infty}{\stackrel{H^{1}\left(\mathbb{R}^{N}\right)}{\longrightarrow}} u$. Let $\varepsilon>0$. Let then $\varepsilon^{\prime}, p_{\varepsilon^{\prime}}$ and $C_{\varepsilon^{\prime}}$ be given by Step 3. By Hölder's inequality, we have for any $v \in H^{1}\left(\mathbb{R}^{N}\right)$,

$$
\int_{\mathbb{R}^{N}}\left|g\left(u_{n}\right)-g(u)\right||v| \mathbb{1}_{A_{n}} \mathrm{~d} x \leqslant\left\|\left(g\left(u_{n}\right)-g(u)\right) \mathbb{1}_{A_{n}}\right\|_{L^{p_{\varepsilon^{\prime}}^{\prime}\left(\mathbb{R}^{N}\right)}}\|v\|_{L^{p^{\prime}}\left(\mathbb{R}^{N}\right)} .
$$

It follows from Sobolev' embedding and (A.18)-(A.19) that,

$$
\sup _{\|v\|_{H^{1}\left(\mathbb{R}^{N}\right)}=1}\left|\left\langle g\left(u_{n}\right)-g(u), v\right\rangle_{H^{-1}\left(\mathbb{R}^{N}\right), H^{1}\left(\mathbb{R}^{N}\right)}\right| \leqslant C\left\|\left(g\left(u_{n}\right)-g(u)\right) \mathbb{1}_{A_{n}}\right\|_{L^{p_{\varepsilon^{\prime}}^{\prime}\left(\mathbb{R}^{N}\right)}}+\varepsilon
$$

We claim that,

$$
\lim _{n \rightarrow \infty}\left\|\left(g\left(u_{n}\right)-g(u)\right) \mathbb{1}_{A_{n}}\right\|_{L^{p_{\varepsilon^{\prime}}^{\prime}\left(\mathbb{R}^{N}\right)}}=0 .
$$

If not, for some $\varepsilon_{0}>0$ and a subsequence, that we will denote by $\left(u_{n}\right)_{n}$, there would exist $h \in$ $L^{p_{\varepsilon^{\prime}}}\left(\mathbb{R}^{N} ; \mathbb{R}\right)$ such that for any $n \in \mathbb{N},\left\|\left(g\left(u_{n}\right)-g(u)\right) \mathbb{1}_{A_{n}}\right\|_{L^{p^{\prime} \varepsilon^{\prime}\left(\mathbb{R}^{N}\right)}} \geqslant \varepsilon_{0},\left|u_{n}\right| \stackrel{\text { a.e }}{\leqslant} h$ and $u_{n} \stackrel{\text { a.e. in } \mathbb{R}^{N}}{n \rightarrow \infty} u$.

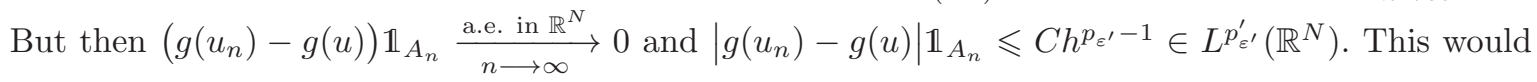
yield to a contradiction by the Lebesgue convergence Theorem. Hence (A.21). It then follows from (A.20)-(A.21) that,

$$
\forall \varepsilon>0, \limsup _{n \rightarrow \infty}\left\|g\left(u_{n}\right)-g(u)\right\|_{H^{-1}\left(\mathbb{R}^{N}\right)} \leqslant \varepsilon .
$$

Letting $\varepsilon \searrow 0$, we get $g \in C\left(H^{1}\left(\mathbb{R}^{N}\right) ; H^{-1}\left(\mathbb{R}^{N}\right)\right)$.

Step 5: Let $\left(u_{n}\right)_{n},\left(v_{n}\right)_{n} \subset H^{1}\left(\mathbb{R}^{N}\right)$ be bounded. If $\lim _{n \rightarrow \infty}\left\|u_{n}-v_{n}\right\|_{L^{p}\left(\mathbb{R}^{N}\right)}=0$, for some $p \in[1, \infty]$, then $\lim _{n \rightarrow \infty}\left|\psi\left(u_{n}\right)-\psi\left(v_{n}\right)\right|=0$.

Let $\varepsilon>0$. For such an $\varepsilon$, let $p_{\varepsilon}$ and $C_{\varepsilon}$ be given by (1.8)-(1.9). Let for any $t \in[0,1], a(t)=$ $\psi\left(v_{n}+t\left(u_{n}-v_{n}\right)\right)$. Then $a \in C^{1}([0,1] ; \mathbb{R})$ and by the mean value Theorem, there exists $t_{n} \in(0,1)$ such that $a(1)-a(0)=a^{\prime}\left(t_{n}\right)(1-0)$, that is

$$
\psi\left(u_{n}\right)-\psi\left(v_{n}\right)=\left\langle g\left(w_{n}\right), u_{n}-v_{n}\right\rangle_{H^{-1}\left(\mathbb{R}^{N}\right), H^{1}\left(\mathbb{R}^{N}\right)} .
$$

where $w_{n}=v_{n}+t_{n}\left(u_{n}-v_{n}\right)$. Note that $\left(w_{n}\right)_{n \in \mathbb{N}}$ is bounded in $H^{1}\left(\mathbb{R}^{N}\right)$. It follows from (1.8)-(1.9), Hölder's inequality and Sobolev's embedding that $\lim _{n \rightarrow \infty}\left\|u_{n}-v_{n}\right\|_{L^{p_{\varepsilon}\left(\mathbb{R}^{N}\right)}}=0$ and

$$
\begin{aligned}
& \left|\psi\left(u_{n}\right)-\psi\left(v_{n}\right)\right| \\
\leqslant & \varepsilon\left(\left\|w_{n}\right\|_{L^{2}\left(\mathbb{R}^{N}\right)}+\kappa\left\|w_{n}\right\|_{L^{2^{\star}}\left(\mathbb{R}^{N}\right)}^{2^{\star}-1}\right)\left\|u_{n}-v_{n}\right\|_{H^{1}\left(\mathbb{R}^{N}\right)}+C_{p_{\varepsilon}}\left\|w_{n}\right\|_{L^{p_{\varepsilon}\left(\mathbb{R}^{N}\right)}}^{p_{\varepsilon}-1}\left\|u_{n}-v_{n}\right\|_{L^{p_{\varepsilon}\left(\mathbb{R}^{N}\right)}} \\
\leqslant & C \varepsilon+C\left\|u_{n}-v_{n}\right\|_{L^{p_{\varepsilon}\left(\mathbb{R}^{N}\right)}} .
\end{aligned}
$$


We infer,

$$
\forall \varepsilon>0, \limsup _{n \rightarrow \infty}\left|\psi\left(u_{n}\right)-\psi\left(v_{n}\right)\right| \leqslant C \varepsilon
$$

from which the result follows.

Step 6: If $u_{n} \stackrel{H_{w}^{1}}{\longrightarrow} u$ then $g\left(u_{n}\right) \stackrel{H_{w}^{-1}}{\longrightarrow} g(u)$.

Since $\left(g\left(u_{n}\right)\right)_{n \in \mathbb{N}}$ is bounded in $H^{-1}\left(\mathbb{R}^{N}\right)$ (Step 1), it is enough to show that $g\left(u_{n}\right) \stackrel{\mathscr{D}^{\prime}\left(\mathbb{R}^{N}\right)}{n \rightarrow \infty} g(u)$. Let

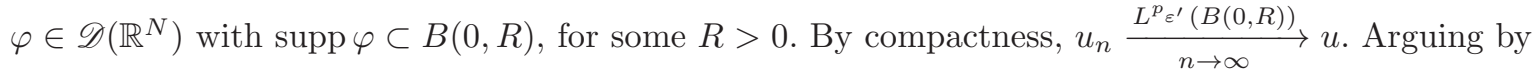
contradiction and using the dominated convergence Theorem, we show in the same way as in Step 4,

$$
\lim _{n \rightarrow \infty} \int_{\mathbb{R}^{N}}\left|g\left(u_{n}\right)-g(u)\right||\varphi| \mathbb{1}_{A_{n}} \mathrm{~d} x=0,
$$

from which we deduce, with help of (A.18),

$$
\forall \varepsilon>0, \limsup _{n \rightarrow \infty}\left|\left\langle g\left(u_{n}\right)-g(u), \varphi\right\rangle_{\mathscr{D}^{\prime}\left(\mathbb{R}^{N}\right) ; \mathscr{D}\left(\mathbb{R}^{N}\right)}\right| \leqslant \varepsilon\|\varphi\|_{H^{1}\left(\mathbb{R}^{N}\right)} .
$$

We conclude as in Step 4.

\section{B Topological vector spaces over the field of complex numbers restricted to the field of real numbers}

Throughout this paper, we consider Banach spaces (or, more generally, complete topological vector spaces) over $\mathbb{R}$ rather than $\mathbb{C}$. The main motivations are the following. Firstly, the linear forms are real-valued and there is a relation of order over $\mathbb{R}$. Secondly, if a function $\psi$ belongs to $C^{1}(X ; \mathbb{R}$ ) (as in Proposition 5.1, for instance), where $X$ is a real Banach space, then $\psi^{\prime} \in C\left(X ; X^{\star}\right)$, where $X^{\star}$ is the $\mathbb{R}$-vector space $\mathscr{L}(X ; \mathbb{R})$. If $X$ is a complex Banach space then $X^{\star}$ is the $\mathbb{C}$-vector space $\mathscr{L}(X ; \mathbb{C})$ and $\psi^{\prime} \in C(X ; \mathscr{L}(X ; \mathbb{R}))$. But then, when a Riesz representation theorem exists, we have two kinds of representation between the elements of $\mathscr{L}(X ; \mathbb{R})$ and those of $X^{\star}=\mathscr{L}(X ; \mathbb{C})$, since $\mathscr{L}(X ; \mathbb{R})$ is not $\mathbb{C}$-linear. On the other hand, if $X$ is a complex Banach space, it could be pleasant to consider $\lambda x$, for $(\lambda, x) \in \mathbb{C} \times X$. So, if $X_{\mathbb{C}}$ is a complex topological vector space, throughout this paper we consider $X_{\mathbb{R}}$ as the elements of $X_{\mathbb{C}}$ over the field $\mathbb{R}$. We then consider the real topological vector space $X_{\mathbb{R}}^{\star}$. For any $(\lambda, x) \in \mathbb{C} \times X, \lambda x \in X_{\mathbb{R}}$, since $X_{\mathbb{R}}$ and $X_{\mathbb{C}}$ have the same elements. In the special case where $H_{\mathbb{C}}$ is a complex Hilbert space whose the inner product is $(., .)_{H}$ then $H_{\mathbb{R}}$ is the real Hilbert space whose the scalar product is $\langle., .\rangle_{H} \stackrel{\text { def }}{=} \operatorname{Re}(., .)_{H}$. In particular, for any $(u, v) \in H_{\mathbb{R}} \times H_{\mathbb{R}}$, $\langle\mathrm{i} u, \mathrm{i} v\rangle_{H}=\langle u, v\rangle_{H}$. Now, assume that $X_{\mathbb{C}}$ is a complex Banach space. Denote by $X_{\mathbb{C}}^{\star}$ and $X_{\mathbb{R}}^{\star}$ the 
topological dual spaces of $X_{\mathbb{C}}$ and $X_{\mathbb{R}}$, respectively. It follows that $X_{\mathbb{C}}^{\star}$ is a $\mathbb{C}$-linear space while $X_{\mathbb{R}}^{\star}$ is only a $\mathbb{R}$-linear space. Let us define the map,

$$
\begin{aligned}
I: X_{\mathbb{C}}^{\star} & \longrightarrow X_{\mathbb{R}}^{\star}, \\
L & \longmapsto \operatorname{Re} L .
\end{aligned}
$$

Then $I$ is a bijective isometry from $X_{\mathbb{C}}^{\star}$ onto $X_{\mathbb{R}}^{\star}$ (Brezis [3, Proposition 11.22, p.361]). With help of this correspondance, we can identify some linear forms. For instance, let $X=L^{p}(\Omega ; \mathbb{C})$, where $\Omega$ is an open subset of $\mathbb{R}^{N}$ and $1 \leqslant p<\infty$. If $p=2$ then the inner and scalar products are given by

$$
(u, v)_{X}=\int_{\Omega} u(x) \overline{v(x)} \mathrm{d} x \quad \text { and } \quad\langle u, v\rangle_{X}=\operatorname{Re} \int_{\Omega} u(x) \overline{v(x)} \mathrm{d} x,
$$

respectively. Using the Riesz representation Theorem for the complex $L^{p}(\Omega ; \mathbb{C})_{\mathbb{C}}$ spaces (Yosida $[15$, Example 3, p.115]) and the bijective isometric map (B.1), it follows that

$$
L^{p}(\Omega ; \mathbb{C})_{\mathbb{R}}^{\star}=L^{p^{\prime}}(\Omega ; \mathbb{C})_{\mathbb{R}},
$$

where $\frac{1}{p}+\frac{1}{p^{\prime}}=1$. More precisely, for any $L \in L^{p}(\Omega ; \mathbb{C})_{\mathbb{R}}^{\star}$, there exists a unique $u \in L^{p^{\prime}}(\Omega ; \mathbb{C})_{\mathbb{R}}$ such that

$$
\langle L, v\rangle_{L^{p}(\Omega)^{\star}, L^{p}(\Omega)}=\operatorname{Re} \int_{\Omega} u(x) \overline{v(x)} \mathrm{d} x,
$$

for any $v \in L^{p}(\Omega ; \mathbb{C})_{\mathbb{R}}$. Furthermore, $\|u\|_{L^{p^{\prime}}(\Omega ; \mathbb{C})_{\mathbb{R}}}=\|L\|_{L^{p}(\Omega ; \mathbb{C})_{\mathbb{R}}^{\star}}$. Finally, we end this appendix with the space of distributions $\mathscr{D}^{\prime}(\Omega ; \mathbb{C})$. We consider the $\mathbb{C}$-complete topological vector space $\mathscr{D}(\Omega ; \mathbb{C})$ restricted to the field $\mathbb{R}$ as above. Then an element $T$ belongs to the $\mathbb{R}$-complete topological vector space $\mathscr{D}^{\prime}(\Omega ; \mathbb{C})$ if $T$ is a $\mathbb{R}$-linear continuous mapping from $\mathscr{D}(\Omega ; \mathbb{C})$ to $\mathbb{R}$. In particular, a function $f \in L_{\text {loc }}^{1}(\Omega ; \mathbb{C})$ (over the field $\left.\mathbb{R}\right)$ defines a distribution $T_{f} \in \mathscr{D}^{\prime}(\Omega ; \mathbb{C})$ by the formula,

$$
\left\langle T_{f}, \varphi\right\rangle_{\mathscr{D}^{\prime}(\Omega ; \mathbb{C}), \mathscr{D}(\Omega ; \mathbb{C})}=\operatorname{Re} \int_{\Omega} f(x) \overline{\varphi(x)} \mathrm{d} x,
$$

for any $\varphi \in \mathscr{D}(\Omega ; \mathbb{C})$. Indeed, $T_{f}$ is clearly a $\mathbb{R}$-linear continuous mapping from $\mathscr{D}(\Omega ; \mathbb{C})$ to $\mathbb{R}$. Furthermore, if $f \in L_{\text {loc }}^{1}(\Omega ; \mathbb{C})$ satisfies,

$$
\operatorname{Re} \int_{\Omega} f(x) \overline{\varphi(x)} \mathrm{d} x=0
$$

for any $\varphi \in \mathscr{D}(\Omega ; \mathbb{C})$, then $f=0$. To see this, we note that $\operatorname{Re}(f), \operatorname{Im}(f) \in L_{\text {loc }}^{1}(\Omega ; \mathbb{R})$ and choosing $\varphi=\psi+\mathrm{i} 0$ and then $\varphi=0+\mathrm{i} \psi$ in the above expression, we get

$$
\int_{\Omega} \operatorname{Re}(f(x)) \psi(x) \mathrm{d} x=\int_{\Omega} \operatorname{Im}(f(x)) \psi(x) \mathrm{d} x=0,
$$


for any $\psi \in \mathscr{D}(\Omega ; \mathbb{R})$. We infer that $\operatorname{Re}(f)=\operatorname{Im}(f)=0$ (Brezis [3, Corollary 4.24, p.110]), from which

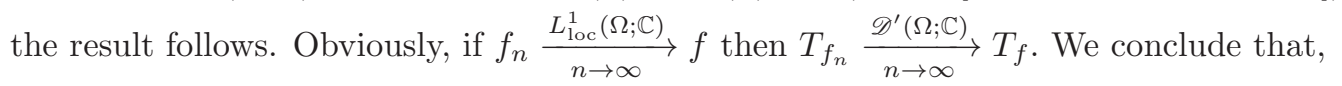

$$
L_{\mathrm{loc}}^{1}(\Omega ; \mathbb{C}) \hookrightarrow \mathscr{D}^{\prime}(\Omega ; \mathbb{C})
$$

with embedding $T: f \in L_{\text {loc }}^{1}(\Omega ; \mathbb{C}) \longmapsto T_{f} \in \mathscr{D}^{\prime}(\Omega ; \mathbb{C})$.

\section{Acknowledgement}

The authors would like to thank the anonymous referees for their valuable feedback and recommendations.

\section{References}

[1] G. Arioli and A. Szulkin. A semilinear Schrödinger equation in the presence of a magnetic field. Arch. Ration. Mech. Anal., 170(4):277-295, 2003.

[2] J. Bergh and J. Löfström. Interpolation spaces. An introduction. Springer-Verlag, Berlin, 1976. Grundlehren der Mathematischen Wissenschaften, No. 223.

[3] H. Brezis. Functional analysis, Sobolev spaces and partial differential equations. Universitext. Springer, New York, 2011.

[4] G. Devillanova and C. Tintarev. Nonlinear Schrödinger equation with bounded magnetic field. J. Differential Equations, 269(10):8998-9025, 2020.

[5] Y. Jabri. The mountain pass theorem, volume 95 of Encyclopedia of Mathematics and its Applications. Cambridge University Press, Cambridge, 2003. Variants, generalizations and some applications.

[6] L. Jeanjean. On the existence of bounded Palais-Smale sequences and application to a Landesman-Lazer-type problem set on $\mathbb{R}^{N}$. Proc. Roy. Soc. Edinburgh Sect. A, 129(4):787-809, 1999.

[7] H. Leinfelder. Gauge invariance of Schrödinger operators and related spectral properties. J. Operator Theory, 9(1):163-179, 1983.

[8] E. H. Lieb and M. Loss. Analysis, volume 14 of Graduate Studies in Mathematics. American Mathematical Society, Providence, RI, second edition, 2001.

[9] M. Schechter and K. Tintarev. Eigenvalues for semilinear boundary value problems. Arch. Rational Mech. Anal., 113(3):197-208, 1990.

[10] I. Schindler. A value function and applications to translation-invariant semilinear elliptic equations on unbounded domains. Differential Integral Equations, 8(4):813-828, 1995.

[11] I. Schindler and K. Tintarev. A nonlinear Schrödinger equation with external magnetic field. Rostock. Math. Kolloq., (56):49-54, 2002.

[12] M. Struwe. Variational methods, volume 34. Springer-Verlag, Berlin, fourth edition, 2008. Applications to nonlinear partial differential equations and Hamiltonian systems. 
[13] K. Tintarev. A relation between critical values and eigenvalues in nonlinear minimax problems. Appl. Anal., 54(1-2):57-71, 1994.

[14] K. Tintarev and K.-H. Fieseler. Concentration compactness. Imperial College Press, London, 2007. Functional-analytic grounds and applications.

[15] K. Yosida. Functional analysis, volume 123 of Grundlehren der Mathematischen Wissenschaften [Fundamental Principles of Mathematical Sciences]. Springer-Verlag, Berlin, sixth edition, 1980. 